\title{
Strategische Optionen der Familien- und Migrationspolitik in Deutschland und Europa
}

\section{Herwig Birg}

\section{Einführung}

Deutschland betreibt seit dem Zweiten Weltkrieg keine Bevölkerungspolitik mehr. Wie tiefgreifend die geschichtliche Zäsur ist, lässt sich daran erkennen, dass der Begriff Bevölkerungspolitik in keinem Gesetz, in keiner Verordnung oder in irgendeinem Text amtlicher Art vorkommt. Das bedeutet jedoch nicht, dass auch die mit diesem Begriff verbundenen Probleme für Deutschland keine Bedeutung mehr hätten.

Wie bei allen menschlichen Handlungen, so müssen auch in der Politik stets Antworten auf folgende Fragen gefunden werden:

1) Von welchem Wollen und welchen Zielen soll sich die Politik leiten lassen?

2) Was steht in der Macht der Politik, welchen Handlungsspielraum hat sie, welche Optionen bieten sich ihr?

3) Welche Bedeutung haben ethische Prinzipien für die Politik, was soll Politik wollen, was verbietet ,sich“ von selbst, was darf sie, was nicht?

Wie kommt es, dass diese Fragen auch für die Bevölkerungsentwicklung in Deutschland von großer Bedeutung sind, obwohl die meisten Repräsentanten unserer Demokratie es bestreiten würden, dass es in Deutschland eine bevölkerungspolitisch wirksame Politik überhaupt gibt? Lässt sich dieser Widerspruch auflösen, indem man die Familienpolitik, die Sozialpolitik oder andere Politikbereiche, deren demographische Auswirkungen insbesondere auf die Geburtenzahl oder auf die Lebenserwartung nicht zu bestreiten sind, begrifflich nicht unter die bevölkerungspolitisch wirksame Politik subsumiert, weil diese Politikbereiche nicht einer bevölkerungspolitischen Intention entspringen, sondern z.B. einer familienpolitischen?

Die Klassifikation einer Politik nach dem Kriterium ihrer Intention ist wichtig, aber für die Beurteilung einer konkreten Politik zählt nicht nur ihre Intention, sondern auch ihre reale Wirkung. Auf die Intention allein kann es nicht ankommen, denn sonst könnte z.B. auch eine Familienpolitik, die mit einer niedrigen Geburtenrate und einer dauernden Bevölkerungsschrumpfung verbunden wäre, völlig zufriedenstellend sein, selbst wenn die Zahl der Familien dadurch ständig abnähme.

Die These, dass es in Deutschland keine Bevölkerungspolitik gibt, weil die Politik hierzulande keine bevölkerungspolitischen Ziele verfolgt und auf keinen dies- 
bezüglichen Intentionen beruht, lässt sich noch weniger aufrecht erhalten, wenn man sie mit der für die demographische Entwicklung besonders folgenreichen Migrationspolitik konfrontiert. Der Begriff Migrations- bzw. Wanderungspolitik kommt zwar ebenso wie der Begriff Bevölkerungspolitik in keinem Gesetz und in keiner Verordnung vor, aber die $\mathrm{Zu}$ - und Abwanderung über die Grenzen Deutschlands wird durch eine Vielzahl von Gesetzen und Verordnungen mit anderen Bezeichnungen und Überschriften geregelt und verwaltet, wenn auch nicht gesteuert, z.B. durch Bestimmungen auf den Gebieten des Staatsangehörigkeitsrechts, des Asyl- und Flüchtlingsrechts, des Ausländer- und Arbeitsrechts und anderen. Der Unterschied zwischen Steuerung und Regelung bzw. Verwaltung ist typisch für die Situation in Deutschland, denn Deutschland ist das einzige Land der Welt mit einem in der Verfassung garantierten Individualrecht auf Asyl. Über die Zahl der Asylbewerber aus einem Land, in dem es politische Verfolgungen gibt, kann nach der Verfassung nicht die deutsche Politik entscheiden, weil die Entscheidung zum Zuzug allein bei den politisch Verfolgten liegt. Deutschland kann regeln, wie es mit den Asylbewerbern umgeht, ihre Zahl kann es jedoch nicht bestimmen. An diesem Grundtatbestand wird und will auch das von der Regierung Schröder initierte Zuwanderungsgesetz nichts ändern.

Das Zuwanderungsgesetz wird vor allem mit wirtschaftlichen Argumenten begründet. Die sinkende Bevölkerungszahl in den jüngeren Altersgruppen soll aus arbeitsmarktpolitischen Gründen durch Zuwanderungen aus dem Ausland kompensiert werden. Eine kompensatorische Zuwanderungspolitik wurde in Deutschland schon seit den 60er Jahren des 20. Jahrhunderts z.B. in Form der Gastarbeiterpolitik betrieben. Mit dem geplanten Zuwanderungsgesetz soll sie systematisiert und intensiviert werden. Dabei sollen aber, wie von offizieller Seite immer wieder betont wird, bevölkerungspolitische Ziele keine Rolle spielen. Die Befürworter des Gesetzes werden zwar nicht müde, die positiven Wirkungen des Zuwanderungsgesetzes auf die Altersstruktur hervor zu heben, eine demographische oder bevölkerungspolitische Wirkung wird dabei aber angeblich nicht angestrebt.

$\mathrm{Zu}$ - und Abwanderungen haben besonders starke demographische Auswirkungen auf die Zahl und Struktur der Bevölkerung, und zwar auch dann, wenn man sie nicht zur Kenntnis nimmt. Die demographischen Wirkungen politischen Handelns (und Unterlassens) können auch in einer Demokratie nicht vermieden, sondern nur anders benannt werden. Aber warum sollte man die bevölkerungspolitischen Auswirkungen der Politik nicht bevölkerungspolitische Auswirkungen nennen? Die Definitionsmacht der Nazis über den Inhalt des Begriffs Bevölkerungspolitik ist mit ihnen untergegangen. Dieses Land muss die Souveränität über seine Sprache wieder gewinnen, ohne die es keine geistige und auf Dauer auch keine politische Souveränität geben kann. Die Demokraten in Deutschland haben nicht nur das Recht, sondern auch die Pflicht, den Begriff Bevölkerungspolitik neu zu definieren und mit einem an demokratischen Zielen orientierten Inhalt zu füllen. 


\section{Biographische und gesellschaftliche Faktoren des Geburten- und Bevölkerungsrückgangs - Folgerungen für die Familienpolitik}

Der Rückgang der Geburtenrate in den letzten Jahrzehnten in Deutschland beruht nach den ÄuBerungen der Befragten aus zahllosen Umfragen nicht auf einer Abschwächung oder gar auf einem Wegfall des Wunsches nach einem Kind, sondern auf wirtschaftlichen und gesellschaftlichen Faktoren, die der Realisierung von Kinderwünschen entgegenstehen. Dabei fällt es jedoch schwer zu klären, was unter dem Wunsch nach einem Kind genau zu verstehen ist, denn die Befragten machen ihre Wünsche von bestimmten Voraussetzungen abhängig, z.B. vom Angebot von Einrichtungen zur Kinderbetreuung, von ausreichenden staatlichen Unterstuitzungszahlungen, vom vorherigen Erreichen bestimmter Ziele der Berufsausbildung und der Erwerbskarriere usw. Ob die Intensität der Kinderwünsche geringer oder die Hürden zu ihrer Verwirklichung höher geworden sind und welchen Anteil die beiden Faktoren am Rückgang der Geburtenrate haben, ist trotz jahrzehntelanger Forschung nicht leicht zu beantworten.

Das bedeutet jedoch nicht, dass unser Wissen zu unvollständig wäre, um die bisherige demographische Entwicklung zu analysieren und darauf aufbauend $\mathrm{Be}$ rechnungen ubber die wahrscheinliche Entwicklung in der Zukunft durchzuführen. Fasst man die Ergebnisse der wissenschaftlichen Forschung úber die Gründe des Ruickgangs der Geburtenrate zusammen, so lassen sich je drei Faktoren auf der Ebene des Individuums und auf der Ebene der Gesellschaft feststellen, aus deren Kombination sich neun Fallgruppen von Ursache-Konstellationen ergeben.

Eine Grundlage für die Beschreibung und Analyse der Ursachen auf der Ebene des Individuums ist der Begriff des biographischen Universums. Dieser Begriff dient zur Bezeichnung der Menge der Lebenslaufalternativen, in denen das Ereignis einer Kindgeburt als ein wesentliches Element des Lebenslaufs mit einer bestimmten Wahrscheinlichkeit auftritt. Die Wahrscheinlichkeit einer Kindgeburt hängt von der Größe und Art des biographischen Universums ab. Größe und Art des biographischen Universums eines Individuums werden von den Faktoren Erziehung und Ausbildung, berufliche Entwicklung und regionaler Lebensraum geformt (Schaubild 1).

Das biographische Universum wird darüber hinaus durch drei weitere Faktoren auf der gesellschaftlichen Ebene bestimmt: Erstens durch das soziale Sicherungssystem, das daruber entscheidet, ob und in welchem Maße die Menschen auf eigene Kinder zur Absicherung der elementaren Lebensrisiken infolge von Alter, Krankheit und Gebrechlichkeit angewiesen sind. Zweitens ist die Frage von Bedeutung, in welchem Maße sich die Frauenerwerbstätigkeit aufgrund der gesellschaftlichen Lebensbedingungen mit der Erziehung von Kindern vereinbaren lässt. Schließlich ist drittens die Frage wichtig, ob in der betreffenden Gesellschaft ein öffentliches Bewusstsein darüber existiert, dass es einen Zusammenhang zwischen der demographischen Struktur einer Gesellschaft und den persönlichen Fortpflan- 
zungsentscheidungen der Individuen gibt. In Analogie zum Begriff des Umweltbewusstseins lässt sich dieses Bewusstsein als Bevölkerungsbewusstsein bezeichnen.

\section{Schaubild 1: Intervenierende Einflussgrößen auf das demographisch- ökonomische Paradoxon}

\begin{tabular}{|c|c|c|c|c|}
\hline & & \multicolumn{3}{|c|}{$\begin{array}{l}\text { Gesellschaftliche Einflussgrößen auf das biographische } \\
\text { Universum }\end{array}$} \\
\hline & & $\begin{array}{l}\text { Soziales Siche- } \\
\text { rungssystem }\end{array}$ & $\begin{array}{l}\text { Vereinbarkeit von } \\
\text { Familien- und } \\
\text { Erwerbsarbeit }\end{array}$ & $\begin{array}{l}\text { Gesellschaftliche } \\
\text { Werte und Priori- } \\
\text { täten für Familien } \\
\text { und Kinder }\end{array}$ \\
\hline \multirow{3}{*}{$\begin{array}{l}\text { Individuelle } \\
\text { Einflussgrößen } \\
\text { auf das } \\
\text { biographische } \\
\text { Universum }\end{array}$} & $\begin{array}{l}\text { Erziehung/ } \\
\text { Ausbildung }\end{array}$ & \multirow{3}{*}{\multicolumn{3}{|c|}{$\begin{array}{l}\text { Demographisch-okonomisches Paradoxon: } \\
\text { Je höher das Pro-Kopf-Einkommen der Frauen, desto } \\
\text { höher sind die okonomischen und biographischen Oppor- } \\
\text { tunitătskosten von Kindern und desto niedriger die Zahl } \\
\text { der Geburten pro Frau. }\end{array}$}} \\
\hline & $\begin{array}{l}\text { Beruf/ } \\
\text { Erwertbsarbeit }\end{array}$ & & & \\
\hline & $\begin{array}{l}\text { Regionale } \\
\text { Lebenswelt }\end{array}$ & & & \\
\hline \multicolumn{5}{|c|}{$\begin{array}{l}\text { Zusätzliche Einflussgrößen auf die Geburtenrate: } \\
\text { - Ausmaß der Einwanderung aus Lảndern mit hoher Geburtenrate } \\
\text { - Ethnische Zusammensetzung der Bevölkerung } \\
\text { - Siedlungsstruktur und Grad der Urbanisierung } \\
\text { - Anteil der kinderlosen Frauen }\end{array}$} \\
\hline
\end{tabular}

Von der Größe und Art des biographischen Universums werden die biographischen Handlungsalternativen und -optionen des Individuums entscheidend beeinflusst. Dabei hat die empirische Lebenslaufforschung gezeigt, dass die Wahrscheinlichkeit einer langfristigen Festlegung im Lebenslauf durch eine Kindgeburt um so geringer ist, je größer die Zahl der Lebenslaufoptionen ist, die aufgrund dieser Festlegung aus dem biographischen Universum ausscheiden würden. ${ }^{1}$ Die ausgeschiedenen Lebenslaufoptionen werden als biographische Opportunitätskosten von Kindern bezeichnet.

Die ökonomischen Opportunitätskosten von Kindern bilden einen Teil der biographischen Opportunitätskosten. Sie lassen sich messen durch die Summe der entgangenen Einkommen, auf die eine Frau verzichten müsste, wenn sie durch die gesellschaftlichen Lebensbedingungen aufgrund einer mangelnden Vereinbarkeit von Erwerbstätigkeit und Familienarbeit nicht erwerbstätig wäre, um Kinder zu erziehen. Mit der biographischen Theorie der Fertilität lässt sich das als ,demographisch-ökonomisches Paradoxon" bezeichnete Phänomen erklären, dass die Zahl der Kinder pro Frau um so mehr zurückging, je stärker das Pro-Kopf-Einkommen zunahm. In Deutschland z.B. ist die Kinderzahl pro Frau heute nur etwa halb so hoch wie in den $60 \mathrm{er}$ Jahren des 20 . Jahrhunderts (1,3 bis 1,4 Kinder pro Frau im

1 H. Birg/E.-J. Flöthmann u. I. Reiter: Biographische Theorie der demographischen Reproduktion. Frankfurt/New York 1991. 
Vergleich zu 2,6 in den 60er Jahren), während gleichzeitig das reale Pro-Kopf-Einkommen auf mehr als das Doppelte stieg.

Das demographisch-ökonomische Paradoxon lässt sich auch in den USA beobachten. Dort liegt die Zahl der Lebendgeborenen mit 2,0 pro Frau (für den Zeitraum 1995-2000) zwar deutlich über dem Durchschnitt aller Industrieländer $(1,6)$, aber der prozentuale Rückgang von dem wesentlich höheren Niveau in den 60er Jahren $(3,3)$ war wie in Deutschland von einem gegenläufigen Anstieg des ProKopf-Einkommens begleitet. Das höhere Niveau der Geburtenrate in den USA beruht vor allem auf den in Schaubild 1 dargestellten gesellschaftlichen Faktoren, darunter auf dem im Vergleich zu Deutschland schlechter ausgebauten sozialen Sicherungssystem. Das durchschnittliche Niveau der Altersrenten auf Grund der kollektiven Rentenversicherung ist z.B. wesentlich niedriger als das Niveau der Renten aus der gesetzlichen Rentenversicherung in Deutschland, so dass die meisten Menschen in den USA im Alter auf eine zusätzliche, privat finanzierte Altersversorgung oder auf die Unterstützung durch ihre Familie angewiesen sind. Das gilt auch für die immer wichtiger werdende Versorgung mit Pflegeleistungen durch Familienmitglieder, denn im Gegensatz zu Deutschland gibt es in den USA keine gesetzliche Pflegeversicherung. Auch im Bereich der Gesundheitsdienste bestehen in den USA große Versorgungslïcken. Etwa 40 Mio. Einwohner haben keine Krankenversicherung.

In modernen Gesellschaften sind die folgenreichsten langfristigen Festlegungen in der Biographie die Festlegungen für einen bestimmten Ausbildungsweg und die anschließende Berufswahl. Diese Entscheidungen stehen am Anfang einer Biographie und fallen oft zeitlich zusammen mit der Entscheidung über die Bindung an einen Partner. Durch diese Festlegungen polarisieren sich die Biographien relativ früh in zwei Gruppen mit und ohne Kinder. Innerhalb der Gruppe mit Kindern ist der Übergang von der Phase ohne Kinder zur Elternschaft mit wesentlich höheren biographischen Opportunitätskosten verbunden als der Übergang vom ersten zum zweiten Kind und vom zweiten zum dritten Kind.

Diese theoretischen Überlegungen lassen sich mit den Daten der Geburtenstatistik und darauf aufbauenden demographisch-biographischen Analysen empirisch stützen. So ist z.B. beim Frauenjahrgang 1955 für die Teilgruppe der Frauen mit drei Kindern die Wahrscheinlichkeit für die Geburt eines vierten Kindes ab dem Alter 32 höher als die Wahrscheinlichkeit für die Geburt eines ersten Kindes bei den noch kinderlosen Frauen dieses Jahrgangs und Alters, und sie ist auch höher als die Wahrscheinlichkeit für die Geburt eines zweiten Kindes bei den Frauen dieses Jahrgangs und Alters, die ein Kind hatten bzw. eines dritten Kindes bei Frauen mit zwei Kindern (Schaubilder 2 und 3). Dieser empirische Befund ist aufgrund der biographischen Fertilitätstheorie zu erwarten. Denn die Theorie besagt, dass die mit einem weiteren Kind aus dem biographischen Universum ausgeschiedenen Lebenslaufoptionen (= biographische Opportunitätskosten) mit jedem zusätzlichen Kind abnehmen.

Nach diesem Befund müsste auch erwartet werden, dass die Wirkung der Familienpolitik auf die Wahrscheinlichkeit der Geburt eines vierten und dritten Kindes 
größer ist als auf die Wahrscheinlichkeit der Geburt eines ersten und zweiten. Auch diese Überlegung lässt sich mit den demographischen Daten bestätigen: In den Schaubildern 2 und 3 ist der Verlauf der Wahrscheinlichkeit für die Geburt eines Kindes in Abhängigkeit vom Alter der Frauen dargestellt. Die im Jahr 1986 eingeführten Maßnahmen der Familienpolitik (Erziehungsgeld und Anrechnung der Erziehungszeiten in der Rentenversicherung der Frauen) führten z.B. bei den Frauen der Jahrgänge 1955 und 1960, die 1986 im Alter 31 bzw. 36 standen, zu einem deutlichen Anstieg der Kurven für die Wahrscheinlichkeit der Geburt von vierten und dritten Kindern, während der Anstieg der Wahrscheinlichkeit für die Geburt zweiter Kinder geringer ausfiel bzw. für erste Kinder gar nicht nachweisbar ist. Der durch die neuen familienpolitischen Maßnahmen von 1986 bewirkte Anstieg der Geburtenwahrscheinlichkeiten ist jedoch insgesamt so gering, dass die Kinderzahl pro Frau z.B. beim Jahrgang 1955 dadurch nur von 1,61 (= Schätzung ohne Berücksichtigung dieser Maßnahmen) auf 1,63 zunahm, was eine Steigerung um lediglich rd. ein Prozent bedeutet. ${ }^{2}$

Entscheidend für die endgültige Zahl der Lebendgeborenen pro Frau ist bei jedem Jahrgang der Anteil der Frauen, die zeitlebens kinderlos bleiben. Der Anteil der Kinderlosen nahm vom Jahrgang 1940 bis zum Jahrgang 1965 von $10,6 \%$ auf $32,1 \%$ zu. Bei der größeren, zwei Drittel und mehr umfassenden Teilgruppe von Frauen, die Kinder hatten, blieb jedoch die Kinderzahl pro Frau mit rd. zwei Kindern von Jahrgang zu Jahrgang relativ konstant. Der Rückgang der Geburtenrate der Jahrgänge ab 1940 beruht also in erster Linie darauf, dass die lebenslange Kinderlosigkeit von Jahrgang zu Jahrgang stieg.

Nach diesen Ergebnissen der demographischen Forschung bieten sich der Familienpolitik zwei Optionen zur Erhöhung der Geburtenrate. Die erste Option hat als Zielgruppe das Drittel der Frauen, die kinderlos bleiben würden. Bei dieser entscheidenden Zielgruppe müsste die lebenslange Kinderlosigkeit deutlich gesenkt werden. Die Zielgruppe für die zweite familienpolitische Option sind die zwei Drittel der Frauen, die Kinder haben. Bei dieser Zielgruppe müsste die durchschnittliche Kinderzahl von rd. zwei Kindern pro Frau auf mehr als zwei erhöht werden.

Die erste Strategie der Verringerung der Kinderlosigkeit hätte - wenn sie erfolgreich wäre - die größte Wirkung auf die Geburtenrate, aber sie bedarf eines familienpolitischen Instrumentariums, das auf diese Zielgruppe zugeschnitten ist. Das entscheidende Element eines solchen familienpolitischen Instrumentariums müsste eine Wertepolitik sein, die die Sinnhaftigkeit eines Lebens mit Kindern als gesellschaftliches Leitbild in der Öffentlichkeit überzeugend vertritt. Ohne eine erfolgreiche Wertepolitik ist damit zu rechnen, dass die biographische Option eines Lebens mit Kindern von einem immer größeren Anteil von Frauen und Männern gar nicht mehr als eine Alternative der Lebenslaufplanung in Erwägung gezogen wird.

2 Zur Berechnung siehe H. Birg: Die demographische Zeitenwende - Der Bevölkerungsrïckgang in Deutschland und Europa. Beck'sche Reihe, Beck Verlag, München 2001, S. 80. 
Schaubild 2: Wahrscheinlichkeit für die Geburt eines ersten, zweiten, dritten bzw. vierten und weiteren Kindes für den Geburtsjahrgang 1955

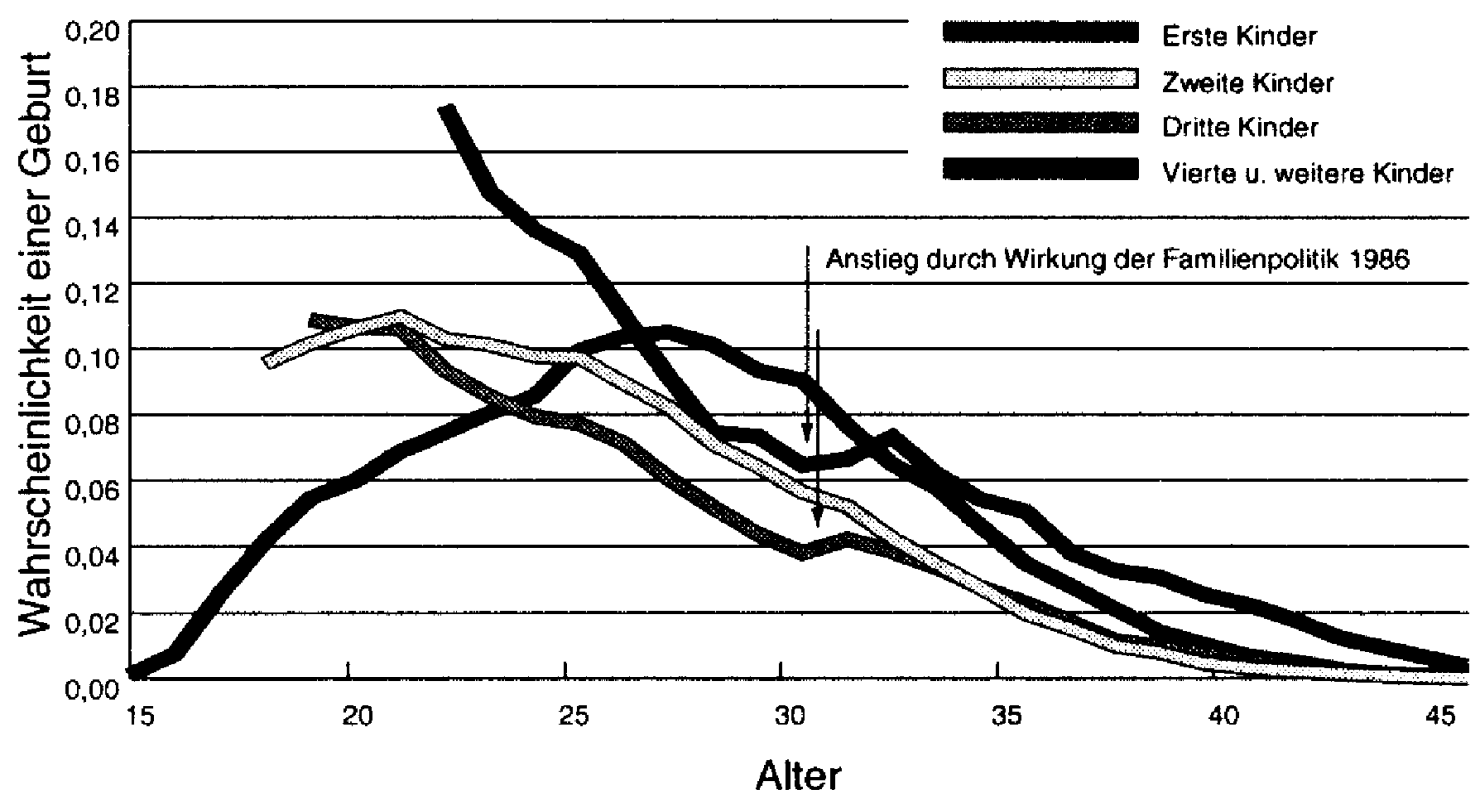

Quelle: Birg/FIothmann, IBS, Univ. Bielefeld 1993

Schaubild 3: Wahrscheinlichkeit für die Geburt eines ersten, zweiten, dritten bzw. vierten und weiteren Kindes für den Geburtsjahrgang 1960

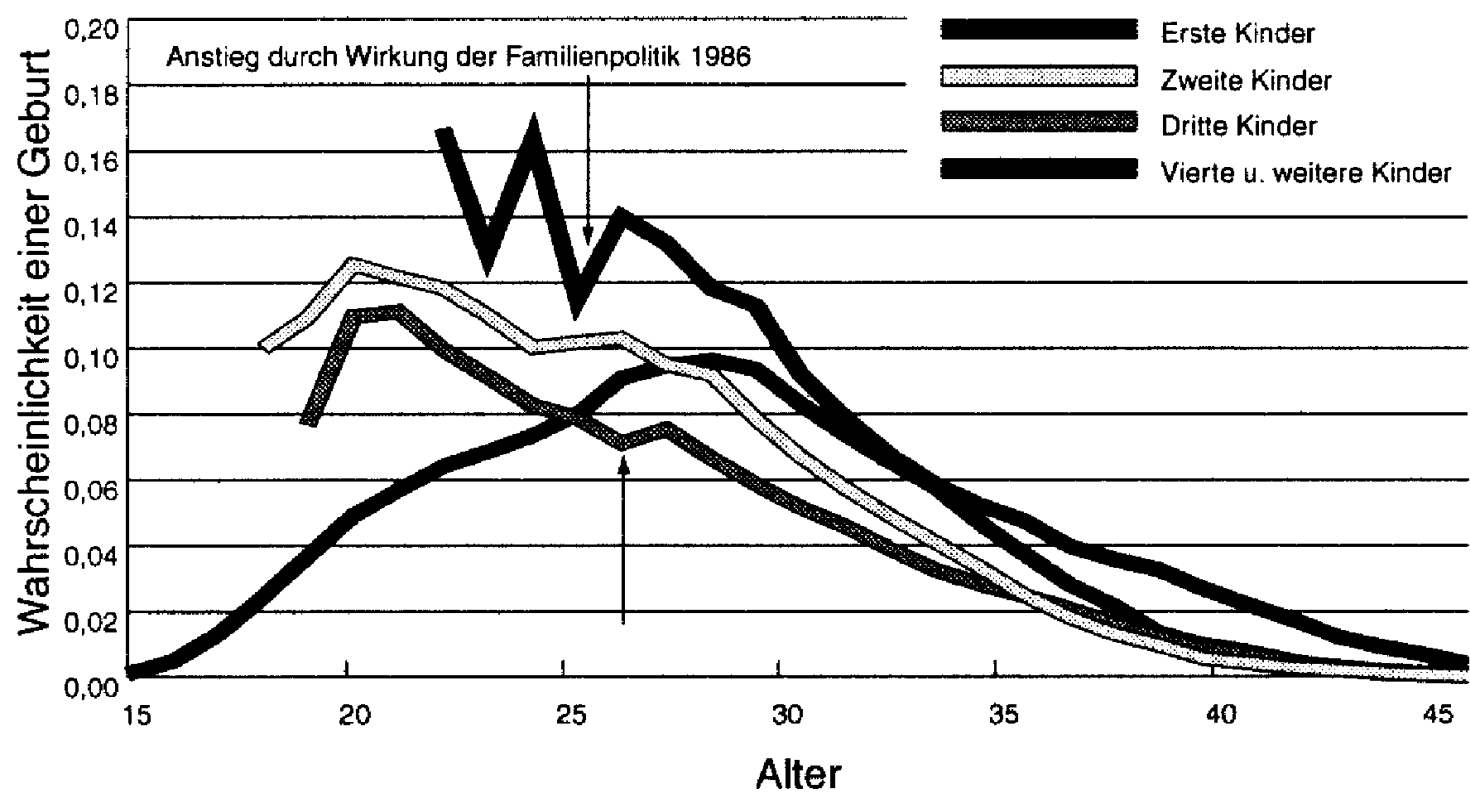

Quelle: Birg/Flöthmann, IBS, Univ. Bielefeld 1993 


\section{Generationenerneuerung durch Geburten oder Generationenersatz durch Migration? Demographische Simulationsrechnungen für die Länder der EU, Japan und die USA}

Die demographische Substanz einer Gesellschaft lässt sich durch zwei prinzipiell verschiedene Strategien bewahren: Die durch Tod ausgeschiedenen Generationen können entweder durch den Nachwuchs erneuert oder sie können durch Einwanderungen aus anderen Gesellschaften ersetzt werden. Für Jahrhunderte galt die erste Variante als die selbstverständliche, natürliche Strategie. Im letzten Viertel des 20. Jahrhunderts ist die Geburtenrate in den meisten Industrieländern jedoch unter das Bestandserhaltungsniveau von 2,13 Lebendgeborenen pro Frau gesunken, so dass die Generationenerneuerung durch Geburten nicht mehr möglich ist. Seitdem wird in einigen Industrieländern die Strategie der Kompensation des Geburtendefizits durch Einwanderungsüberschüsse als Standardmodell der demographischen Entwicklung betrachtet und als politische Strategie umgesetzt.

Der Übergang der Politik von der Erneuerungsstrategie durch Geburten zur Kompensationsstrategie mittels Wanderungen wurde in keinem Land durch öffentliche Debatten vorbereitet und durch demokratische Entscheidungen eingeleitet, sondern stillschweigend und mehr oder weniger unreflektiert vollzogen. Eine Ausnahme von dieser Praxis der Stummheit bildet der von der Bevölkerungsabteilung der Vereinten Nationen im März 2000 veröffentlichte Forschungsbericht mit dem Titel: „Replacement Migration"."3

Der Titel des Forschungsberichts ist programmatisch. Der Begriff ,replacement migration" tritt an die Seite, wenn nicht sogar an die Stelle des bisher gebräuchlichen Begriffs „replacement fertility“. Die analoge sprachliche Konstruktion der beiden Begriffe soll ihre inhaltliche Symmetrie unterstreichen. Die beiden Strategien zur Wahrung der demographischen Substanz werden dabei als prinzipiell gleichwertige Mittel zur Erreichung des gleichen Ziels dargestellt. Im Untertitel des Forschungsberichts wird zwar hinter die Nennung des Ziels ein Fragezeichen gesetzt, aber der Bericht liest sich in weiten Teilen so, als stünde an der Stelle des Fragezeichens ein Ausrufungszeichen: „Is (replacement migration) a solution to declining and ageing populations?"

Für 8 Länder sowie für Europa und die Europäische Gemeinschaft beantwortet der Bericht folgende fünf Fragen:

1) Wie würde sich die Bevölkerung bis 2050 unter mittleren Annahmen zur Fertilität, Mortalität und Migration entwickeln?

2) Wie würde sie sich unter mittleren Annahmen zur Fertilităt und Mortalität entwickeln, wenn unterstellt wird, dass keine Wanderungen stattfinden?

3 UN (Ed.): Replacement migration: Is it a solution to declining and ageing populations? Population Division, Dept. of Economic and Social Affairs, United Nations Secretariat, New York 2000. 
3) Wieviel Wanderungen sind für eine konstante Bevölkerungszahl erforderlich?

4) Wieviel Wanderungen sind für eine konstante Erwerbspersonenzahl (= Altersgruppe 15-64) erforderlich?

5) Wieviel Wanderungen werden für eine konstante Altersstruktur benötigt (konstanter Altenquotient $=$ Relation aus der Zahl der über 65-Jährigen zu den 15bis 64-Jährigen)?

In Deutschland hat vor allem die Antwort auf die fünfte Frage Aufsehen erregt: Bis 2050 müssten 188 Mio. mehr Menschen nach Deutschland einwandern als auswandern, wenn der Altenquotient konstant bleiben soll. Dadurch würde sich die Bevölkerungszahl von 82 Mio. auf 299 Mio. erhöhen. Exorbitante Zuwanderungssalden ergeben sich auch für England (60 Mio.), Frankreich (94 Mio.), Italien (120 Mio.), Russland (257 Mio.), die USA (593 Mio.) bzw. für die Europäische Union (1.386 Mio.). Die durchschnittlichen jährlichen Einwanderungssalden sind entsprechend hoch, sie betragen für Deutschland z.B. 3,4 Mio. pro Jahr (Tabelle 1).

Die in dem Forschungsbericht enthaltenen Antworten auf die fünf Fragen sind zum Teil spektakulär. Dies führte in der Öffentlichkeit zu dem falschen Eindruck, als ob der innovative Charakter dieser Untersuchung in ihren Antworten läge. Das Besondere ist jedoch die neuartige Fragestellung. Seit der Veröffentlichung des UN-Berichts steht neben dem klassischen Begriff der „replacement fertility“ der neue Begriff ,replacement migration“, als ob die beiden Begriffe immer schon für denselben Zweck im Gebrauch gewesen wären und eine Einheit gebildet hätten. Die beiden Begriffe fungieren in dem UN-Bericht als eine Art Doppelbegriff, der das veränderte Denken über die demographische Zukunft der Industrieländer widerspiegelt. Der Leser muss den Eindruck gewinnen, als ob sich mit der Einführung des Begriffs ,replacement migration“ auch der Handlungsspielraum der Politik durch neue Perspektiven und Optionen erweitert hätte.

Wer diesen Bericht in diesem Sinne interpretiert, unterliegt jedoch einem gravierenden Irrtum. Denn diese Studie liefert einen weiteren Beleg dafür, dass der Übergang von der natürlichen Strategie der Erneuerung durch Geburten zur Strategie der Kompensation durch Einwanderungen die zunehmende Alterung als zentrales Hauptproblem der demographischen Entwicklung nicht verhindern, sondern allenfalls dämpfen kann ${ }^{4}$. Für Deutschland z.B. ergibt sich aus dem Bericht für den Fall ohne Wanderungen eine Erhöhung des Altenquotienten (65+/15-64) von 1995 bis 2050 von 22,7 auf 57,1. Für den Fall eines Einwanderungssaldos von rd. 200 Tsd. jüngeren Menschen pro Jahr ist der Altenquotient im Jahr 2050 nicht wesentlich niedriger, er beträgt 48,8 statt 57,1. Für die Europäische Union insgesamt, für die ein vergleichsweise niedriger Wanderungssaldo von 297 Tsd. angenommen

4 Für Deutschland liegen entsprechende Untersuchungen schon länger vor, z.B. von H. Birg, E.-J. Flöthmann, Th. Frein, K. Ströker: Simulationsrechnungen zur Bevölkerungsentwicklung in den alten und neuen Bundesländern im 21. Jahrhundert. Materialien des Instituts für Bevölkerungsforschung und Sozialpolitik, Bd. 45, Universität Bielefeld, Bielefeld 1998. 
Tabelle 1: Wanderungssaldo 1995-2050 nach Szenario und Ländern bzw. Regionen (in Tausend)

\begin{tabular}{|c|c|c|c|c|c|}
\hline Szenario & $\mathbf{I}$ & H & III & IV & $\mathbf{V}$ \\
\hline $\begin{array}{l}\text { Land bzw. } \\
\text { Region }\end{array}$ & $\begin{array}{l}\text { Mittlere } \\
\text { variante }\end{array}$ & $\begin{array}{l}\text { Mittlere Vari- } \\
\text { ante ohne } \\
\text { Wanderungen }\end{array}$ & $\begin{array}{l}\text { konstante } \\
\text { Bevölkerungs- } \\
\text { zahl }\end{array}$ & $\begin{array}{l}\text { konstante } \\
\text { Altersgruppe } \\
15-54\end{array}$ & $\begin{array}{l}\text { konstante } \\
\text { Relation } \\
15-64 / 65+\end{array}$ \\
\hline \multicolumn{6}{|c|}{ A. Gesamtzahl } \\
\hline Frankreich & 525 & 0 & 1473 & 5459 & 93794 \\
\hline Deutschland & 11400 & 0 & 17838 & 25209 & 188497 \\
\hline Italien & 660 & 0 & 12944 & 19610 & 119684 \\
\hline Japan & 0 & 0 & 17141 & 33487 & 553495 \\
\hline $\begin{array}{l}\text { Russische } \\
\text { Föderation }\end{array}$ & 7417 & 0 & 27952 & 35756 & 257110 \\
\hline $\begin{array}{l}\text { Großbritan- } \\
\text { nien }\end{array}$ & 1200 & 0 & 2634 & 6247 & 59775 \\
\hline $\begin{array}{l}\text { Vereinigte } \\
\text { Staaten }\end{array}$ & 41800 & 0 & 6384 & 17967 & 592757 \\
\hline Europa & 23530 & 0 & 100137 & 161346 & 1386151 \\
\hline $\begin{array}{l}\text { Europäische } \\
\text { Union }\end{array}$ & 16361 & 0 & 47456 & 79605 & 700506 \\
\hline \multicolumn{6}{|c|}{ B. Durchschnitt pro Jahr } \\
\hline Frankreich & 10 & 0 & 27 & 99 & 1705 \\
\hline Deutschland & 207 & 0 & 324 & 458 & 3427 \\
\hline Italien & 12 & 0 & 235 & 357 & 2176 \\
\hline Japan & 0 & 0 & 312 & 609 & 10064 \\
\hline $\begin{array}{l}\text { Russische } \\
\text { Föderation }\end{array}$ & 135 & 0 & 508 & 650 & 4675 \\
\hline $\begin{array}{l}\text { Großbritan- } \\
\text { nien }\end{array}$ & 22 & 0 & 48 & 114 & 1087 \\
\hline $\begin{array}{l}\text { Vereinigte } \\
\text { Staaten }\end{array}$ & 760 & 0 & 116 & 327 & 10777 \\
\hline Europa & 428 & 0 & 1821 & 2934 & 25203 \\
\hline $\begin{array}{l}\text { Europăische } \\
\text { Union }\end{array}$ & 297 & 0 & 863 & 1447 & 12736 \\
\hline
\end{tabular}

wurde, ist der Unterschied entsprechend geringer $(52,9$ versus 51,0$)$, ebenso für die USA mit einem angenommenen Wanderungssaldo von 760 Tsd. pro Jahr (38,9 versus 35,0 ). Aus diesen Zahlen des UN-Berichts ergibt sich folgendes Fazit: Wollte man die demographische Alterung, die in erster Linie auf der niedrigen Geburtenrate und erst in zweiter Linie auf der zunehmenden Lebenserwartung beruht, durch die Einwanderungen jüngerer Menschen verhindern, wären dafür so hohe Einwan- 
derungszahlen erforderlich, dass dadurch mehr Probleme geschaffen als gelöst würden.

\section{Demographische Projektionsrechnungen für die Länder der Europäischen Union}

Die folgenden Berechnungen für die 15 Länder der Europäischen Union wurden am Institut für Bevölkerungsforschung und Sozialpolitik der Universität Bielefeld durchgeführt. Sie erstrecken sich auf den Zeitraum 1998-2100. Im folgenden wird vor allem über die Ergebnisse der Variante ohne Wanderungen berichtet. Die Variante unter Einschluss von Wanderungen wurde von Thomas Frein als Diplomarbeit an meinem Lehrstuhl durchgeführt, sie ist bislang nicht veröffentlicht. Die Ergebnisse sind jedoch detailliert dokumentiert und stehen der Fachwelt zur Verfügung. Die Darstellung der Entwicklung in den EU-Ländern unter Einschluss von Wanderungen stützt sich hier auf die in dem zitierten UN-Bericht „Replacement Migration" veröffentlichten Daten.

Im Jahr 1998 betrug die Zahl der Lebendgeborenen pro Frau im Durchschnitt der 15 EU-Länder 1,47. In den nördlichen Ländern (Irland, Vereinigtes Königreich, Finnland, Schweden und Dänemark) waren es 1,70, in Mitteleuropa 1,41 (Deutschland, Niederlande, Belgien, Luxemburg und Österreich) und in Südeuropa 1,39 (Frankreich, Italien, Spanien, Portugal und Griechenland). Für die einzelnen Länder sind die entsprechenden Zahlen in Tabelle 2 dargestellt.

Die Projektionsrechnungen beruhen auf folgenden Annahmen:

1) Die Zahl der Lebendgeborenen pro Frau bleibt auf dem Niveau von 1998 konstant bzw. nimmt leicht zu. Der Durchschnitt der 15 Länder erhöht sich annahmegemäß von 1998 bis 2050 von 1,47 auf 1,51 .

2) Die in den Ländern geringfügig differierende Lebenserwartung steigt von 1998 bis $2080 \mathrm{im}$ Durchschnitt aller 15 Länder bei den Männern von 74,53 auf 80,00 und bei den Frauen von 80,79 auf 86,25.

3) Die Annahmen für die Wanderungssalden beruhen auf differenzierten Analysen der Migrationsströme zwischen den 15 Ländern untereinander und zwischen den 15 Ländern gegenüber den Ländern außerhalb der EU.

Auf diese Annahmen soll hier nicht näher eingegangen werden, weil für die Darstellung der Entwicklung einschlieBlich Wanderungen die Ergebnisse des einfacheren Vorausschätzungsmodells der Population Division der UN verwendet werden (UN, Replacement Migration).

Die Projektionsrechnungen sind keine Prognosen oder Prophezeiungen, sondern bedingte Wenn-Dann-Aussagen über die Zukunft, deren Ergebnisse genau bzw. näherungsweise eintreffen, wenn die Annahmen exakt bzw. annähernd richtig gesetzt werden. Die Ergebnisse lassen sich in 6 Punkten zusammenfassen:

1) Die Bevölkerungszahl der EU nimmt von 1998 bis 2050 (ohne Beruicksichtigung von Wanderungen) von 375 Mio. auf 296 Mio. und bis 2100 auf 184 Mio. ab. Der prozentuale Bevölkerungsrückgang ist in Italien, Spanien und 
Tabelle 2: Lebendgeborene pro Frau und Altenquotient in den Ländern der Europäischen Union 1998 and 2050 (ohne Wanderungen)

\begin{tabular}{|c|c|c|c|c|c|}
\hline & \multirow{2}{*}{$\begin{array}{l}\text { Lebend- } \\
\text { geborene } \\
\text { pro Frau (TFR) } \\
1998\end{array}$} & \multicolumn{2}{|c|}{ Altenquotient (1) } & \multicolumn{2}{|c|}{ Altenquotient (2) } \\
\hline & & 1998 & 2050 & 1998 & 2050 \\
\hline Nordeuropa & 1,70 & 37,2 & 69,4 & 23,7 & 44,5 \\
\hline Großbritannien & 1,72 & 37,6 & 69,3 & 23,9 & 44,4 \\
\hline Dänemark & 1,72 & 34,7 & 65,0 & 22,2 & 42,7 \\
\hline Schweden & 1,50 & 41,2 & 78,7 & 27,1 & 49,7 \\
\hline Finnland & 1,70 & 35,2 & 68,8 & 22,0 & 44,5 \\
\hline Irland & 1,93 & 28,3 & 61,3 & 17,0 & 39,4 \\
\hline Mitteleuropa & $\overline{1,41}$ & 38,3 & 86,3 & 23,0 & $\overline{55,9}$ \\
\hline Deutschland & 1,36 & 39,8 & 90,7 & 23,3 & 58,5 \\
\hline Österreich & 1,34 & 34,8 & 90,6 & 22,9 & 59,8 \\
\hline Belgien & 1,55 & 40,0 & 77,2 & 25,3 & 50,3 \\
\hline Niederlande & 1,63 & 31,3 & 72,8 & 19,9 & 47,6 \\
\hline Luxemburg & 1,68 & 33,7 & 68,7 & 21,3 & 44,8 \\
\hline Südeuropa & 1,39 & 39,4 & 87,8 & 24,7 & 58,8 \\
\hline Frankreich & 1,75 & 37,9 & 69,7 & 24,1 & 45,1 \\
\hline Italien & 1,18 & 41,6 & 103,8 & 26,1 & 70,3 \\
\hline Spanien & 1,18 & 38,2 & 105,6 & 24,1 & 72,6 \\
\hline Portugal & 1,46 & 36,9 & 80,2 & 22,4 & 54,4 \\
\hline Griechenland & 1,29 & 41,7 & 98,4 & 25,0 & 66,5 \\
\hline EU insgesamt & $\overline{1,47}$ & 38,6 & 82,8 & 24,0 & $\overline{54,2}$ \\
\hline \multicolumn{6}{|c|}{ Altenquotient 1: $(\mathrm{P}(60+) / \mathrm{P}(20-60))$ 100; Altenquotient 2: $(\mathrm{P}(65+) / \mathrm{P}(15-65)) 100$} \\
\hline
\end{tabular}

Deutschland stärker als im Durchschnitt der EU, in Frankreich und England schwächer. Aufgrund der hohen Geburtenrate und der günstigeren Altersstruktur haben Frankreich und England auch ohne Einwanderungsüberschüsse noch bis 2015 leichte Bevölkerungszuwächse, Irland noch bis 2050, bevor auch dort die Schrumpfung einsetzt (Tabelle 2, Schaubild 4).

2) Die Bevölkerungsschrumpfung beruht auf einer Abnahme der Zahl der Jungen bei einer gleichzeitigen Zunahme der Zahl der Älteren: Die Bevölkerungspyramide der EU verändert ihre Form und wird zu einem Pilz, wobei die Altersgruppe 70-80 die am stärksten besetzte Altersgruppe ist (Schaubild 5).

3) Die demographische Alterung ist eine automatische Folge der Bevölkerungsschrumpfung. Sie lässt sich durch den Altenquotienten und seine Veränderung messen. Der Altenquotient wird in der Literatur auf zweierlei Art definiert: $\mathrm{Al}$ - 
tenquotient $1=$ Zahl der über 60 -Jährigen und älteren auf 100 Menschen im Alter von 20 bis 60 . Altenquotient $2=$ Zahl der über 65 -Jährigen und älteren auf 100 Menschen im Alter von 15 bis unter 65. Die beiden Altenquotienten sind in gleicher Weise für die Beschreibung der demographischen Alterung geeignet, sie sind hoch miteinander korreliert (Schaubild 6). Der Altenquotient 1 betrug 1998 im Mittel der 15 EU-Länder 38,57, er steigt bis 2050 auf 82,75. Der Altenquotient 2 betrug 1998 23,96, er steigt bis 2050 auf 54,24. Beide Altenquotienten nehmen um den gleichen Faktor 2,26 zu.

4) Die international vergleichende Analyse für die 15 Länder der EU ergibt einen gegenläufigen Zusammenhang zwischen der Höhe der Geburtenrate und der Intensität der demographischen Alterung: Je höher die Zahl der Lebendgeborenen pro Frau, desto niedriger ist der Altenquotient in der Zukunft. Die niedrigste Geburtenrate bzw. den höchsten Altenquotienten in der Zukunft haben Spanien, Italien und Griechenland. Die höchste Geburtenrate und den niedrigsten Altenquotienten haben Irland, Dänemark, Frankreich, Großbritannien und Finnland (Schaubild 7).

5) Die Einwanderung jüngerer Menschen würde den Anstieg des Altenquotienten in der EU nur geringfügig mildern. Auch in den USA hat die Einwanderung junger Menschen nur einen relativ geringen Einfluss auf den Anstieg des Altenquotienten. Nach Berechnungen der UN-Population Division erhöht sich der Altenquotient 2 in der EU von 1998 bis 2050 ohne Wanderungen von 23,2 auf 52,9 und mit Wanderungen auf 51,0. Für Deutschland steigt der Altenquotient ohne Wanderungen von 22,7 auf 57,1 und mit Wanderungen auf 48,8. Für die USA wurde ein Anstieg von 19,2 auf 38,9 (ohne Wanderungen) bzw. auf 35,0 (mit Wanderungen) berechnet (Schaubild 8).

6) Die für das Angebot an Arbeitsplätzen wichtige Altersgruppe 15-65 hat in den Ländern mit überdurchschnittlicher Geburtenrate auch ohne Wanderungen noch bis 2005-2010 leichte und im Falle von Irland bis 2040-2045 noch relativ starke Zuwächse. Zur Ländergruppe mit Zuwächsen gehören neben Irland die Länder Frankreich, Großbritannien, Holland, Dänemark und Finnland. In den übrigen Ländern sowie im Durchschnitt der EU schrumpft diese Altersgruppe bereits ab 1998 (Schaubild 9). Im Unterschied zur Altersgruppe 16-65 schrumpft das jüngere Erwerbspersonenpotential in der Altersgruppe 20-40 mit Ausnahme von Irland in sämtlichen Ländern schon ab 1998. Besonders stark ist die Schrumpfung in Italien und Spanien (bis 2050 minus $47 \%$ ) sowie in Griechenland, Deutschland und Österreich (bis 2050 minus $40 \%$ ) (Schaubild (0).

Fazit: In den Ländern der Europäischen Union streuten die Geburtenraten 1998 in einem relativ breiten Intervall von rd. 1,2 Lebendgeborenen pro Frau in Spanien und Italien bis zu 1,7 in Irland, Großbritannien und Frankreich. Je niedriger die Geburtenrate ist, desto intensiver ist die demographische Alterung in den nächsten Jahrzehnten und desto stärker die Bevölkerungsschrumpfung und die Abnahme des Erwerbspersonenpotentials. 
Schaubild 4: Bevölkerungsentwicklung im 21. Jahrhundert in Ländern der EU (15), der Türkei und in den südlichen Anrainerstaaten des Mittelmeeres

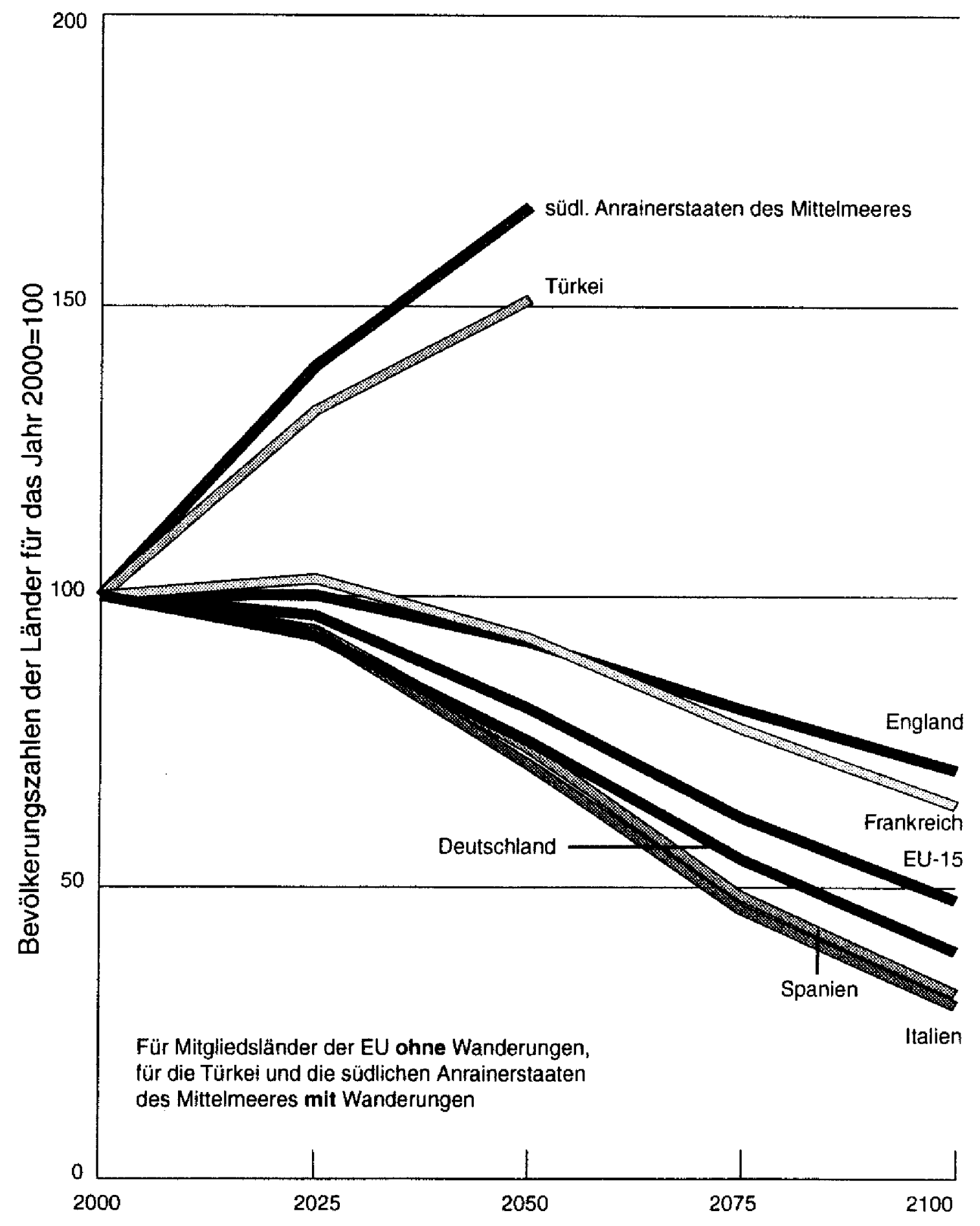

Quelle: H. Birg, IBS, Universităt Bielefeld.

Daten: für Deutschland: H. Birg U. E.-J. Flöthmann: Demographische Projektionsrechnungen für die Rentenretorm 2000, IBS-Materialien, Bd. 47A, Var. 1, S. 130;

für die übrigen EU-Mitgliedsländer: Th. Frein: Unveróffentlichte Projektionsrechnungen; für die Türkei und die südlichen Anrainerstaaten des Mittelmeeres: UN (Ed.): World Population Prospects, 1998 Revision, New York 1999 (mittlere Projektionsvariante). 
Schaubild 5: Altersstruktur der Bevölkerung der EU in den Jahren 2000, 2025, 2050, 2075 und 2100
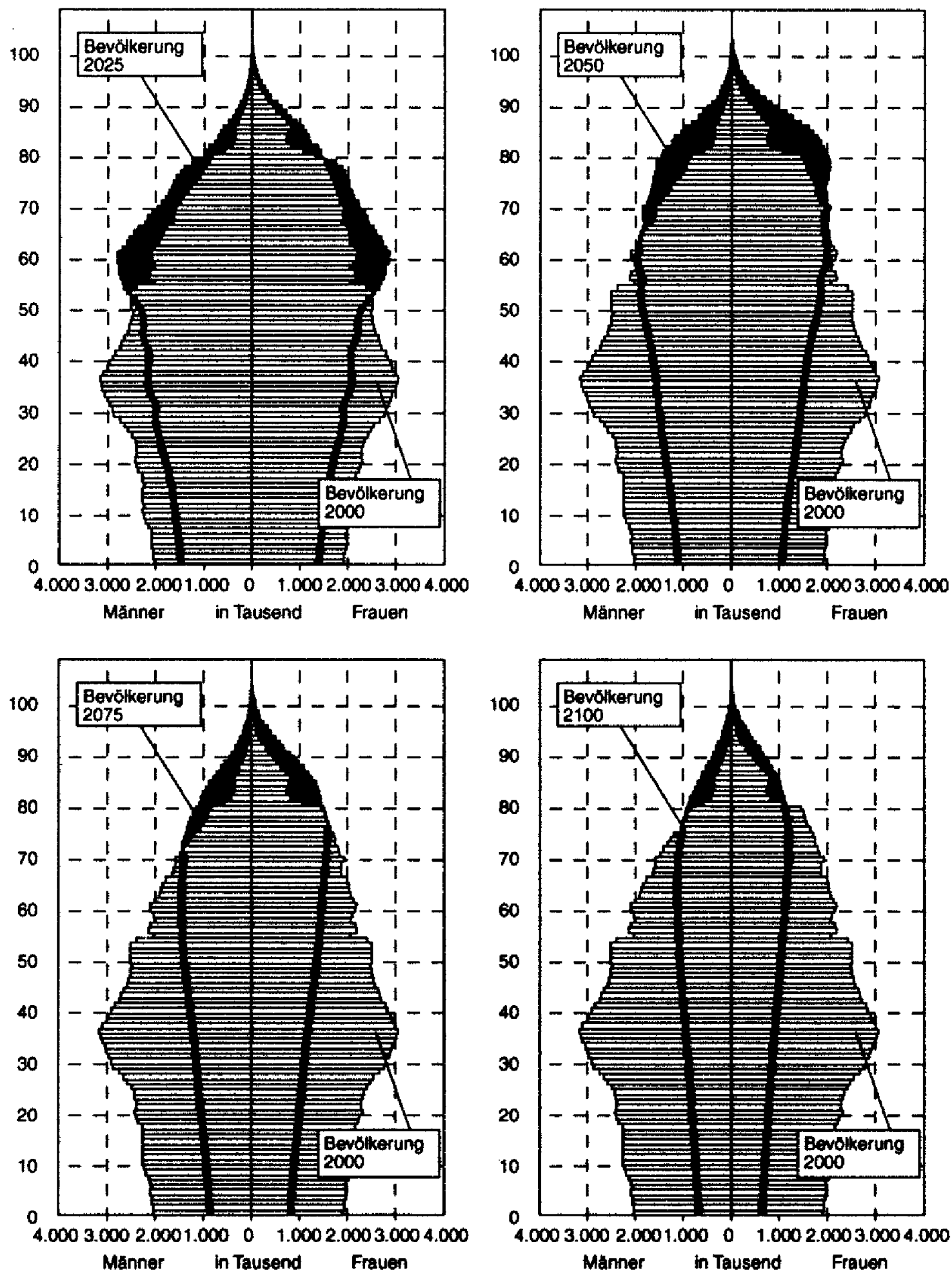

Quelle: Th. Frein, unveroffentlichte Projektionsrechnungen 2000 ohne Wanderungen 
Schaubild 6: Der Altenquotient in den Ländern der Europäischen Union 1998 - nach zwei unterschiedlichen Definitionen

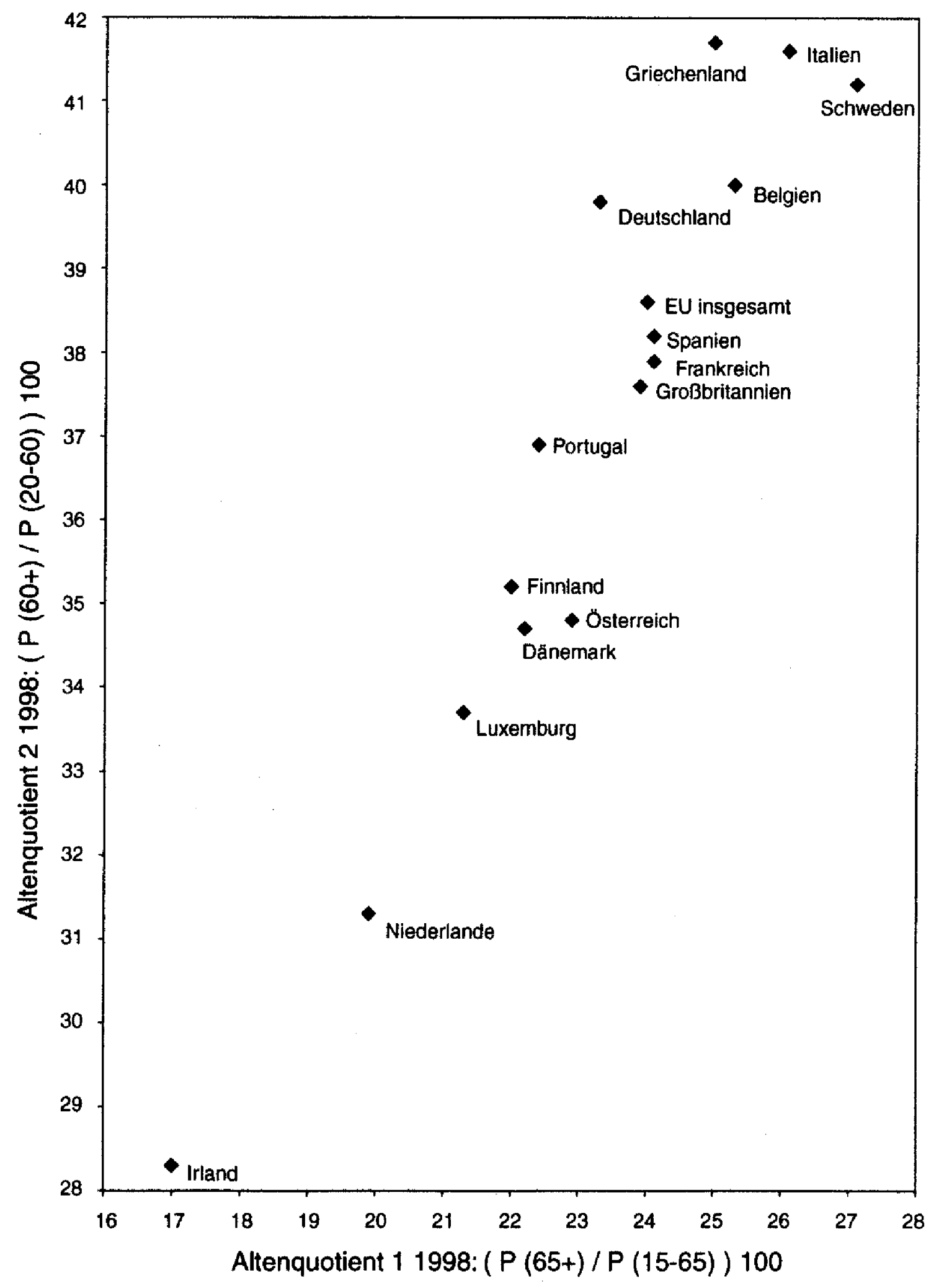

Quelle: H. Birg, IBS, Universitäł Bielefeld 
Schaubild 7: Der gegenläufige Zusammenhang zwischen der Höhe der Kinderzahl pro Frau und der Intensität der demographischen Alterung Vorausberechnungen für die Länder der Europäischen Union ohne Wanderungen

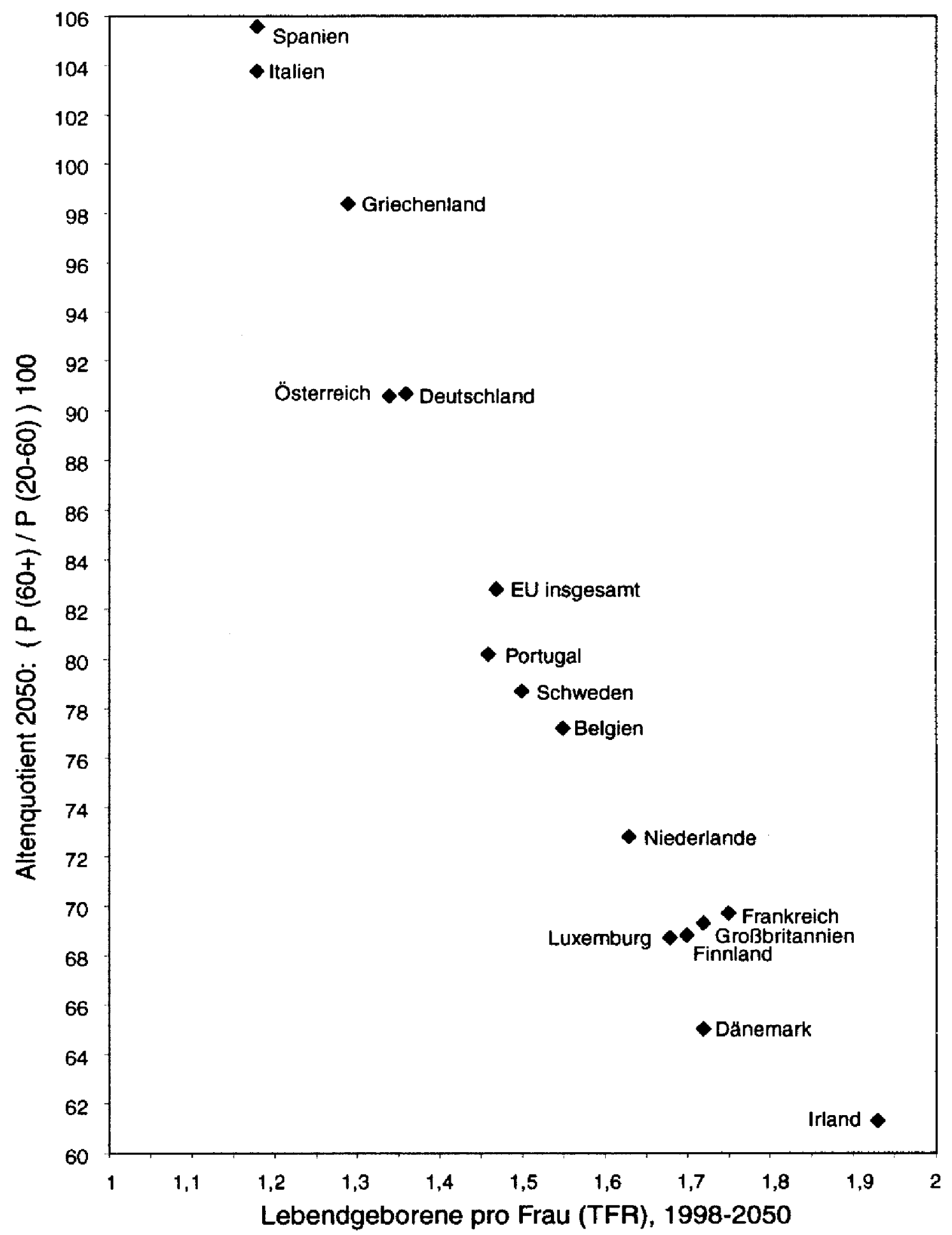

Quelle: H. Birg, IBS, Universität Bielefeld. 
Schaubild 8: Projektionsrechnungen zum Anstieg des Altenquotienten in den Industrieländern (1995-2050)

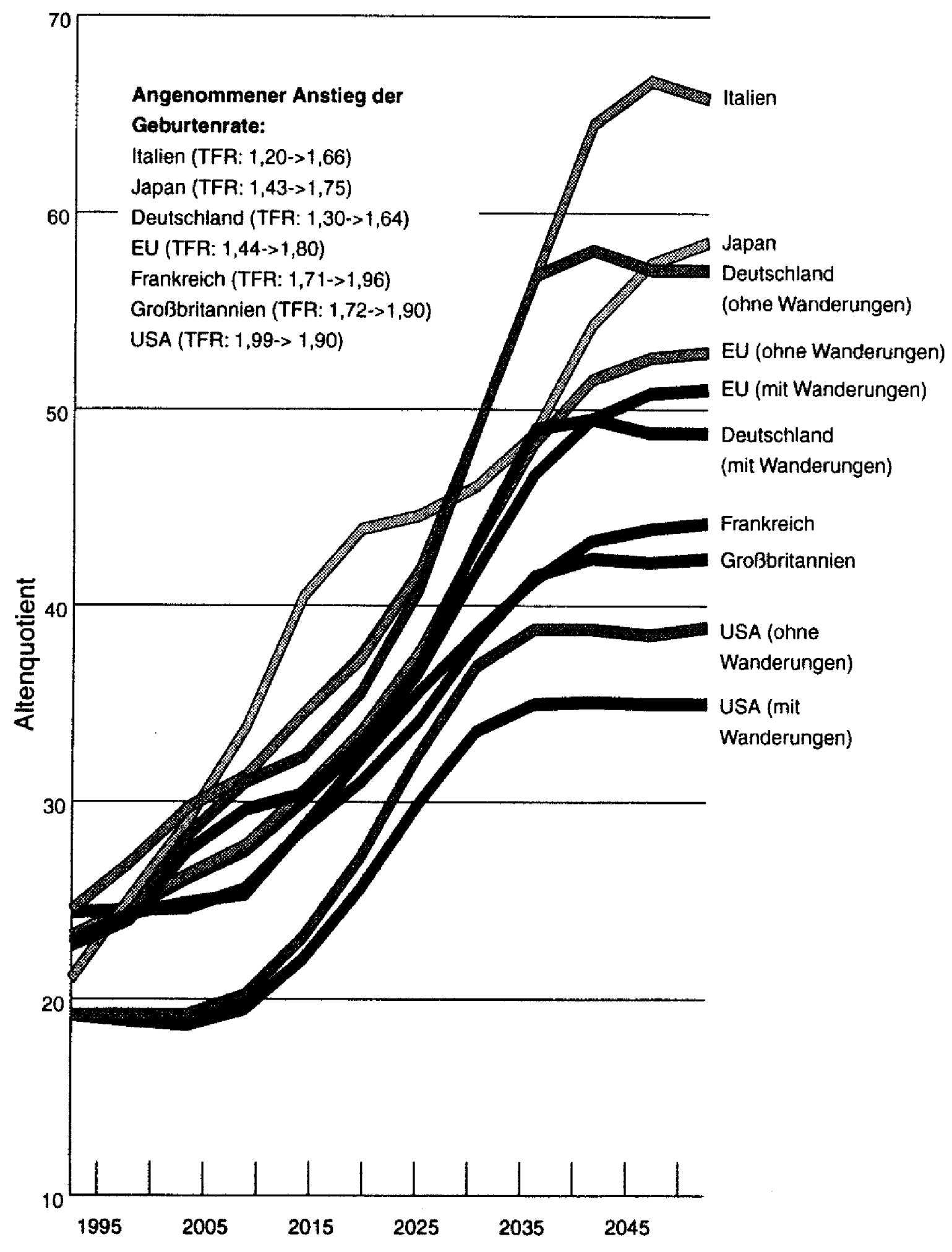

Queile: H. Blrg; Datenbasis: UN, Replacement migration, medium varkant, New York 2000.

1. Altenquotient $=(P(65+) / P(15-64)) * 100$; 
Schaubild 9: Projektion der Zahl der Personen im erwerbsfähigen Alter (15-65) in den Ländern der EU, 1998-2050 (ohne Wanderungen)

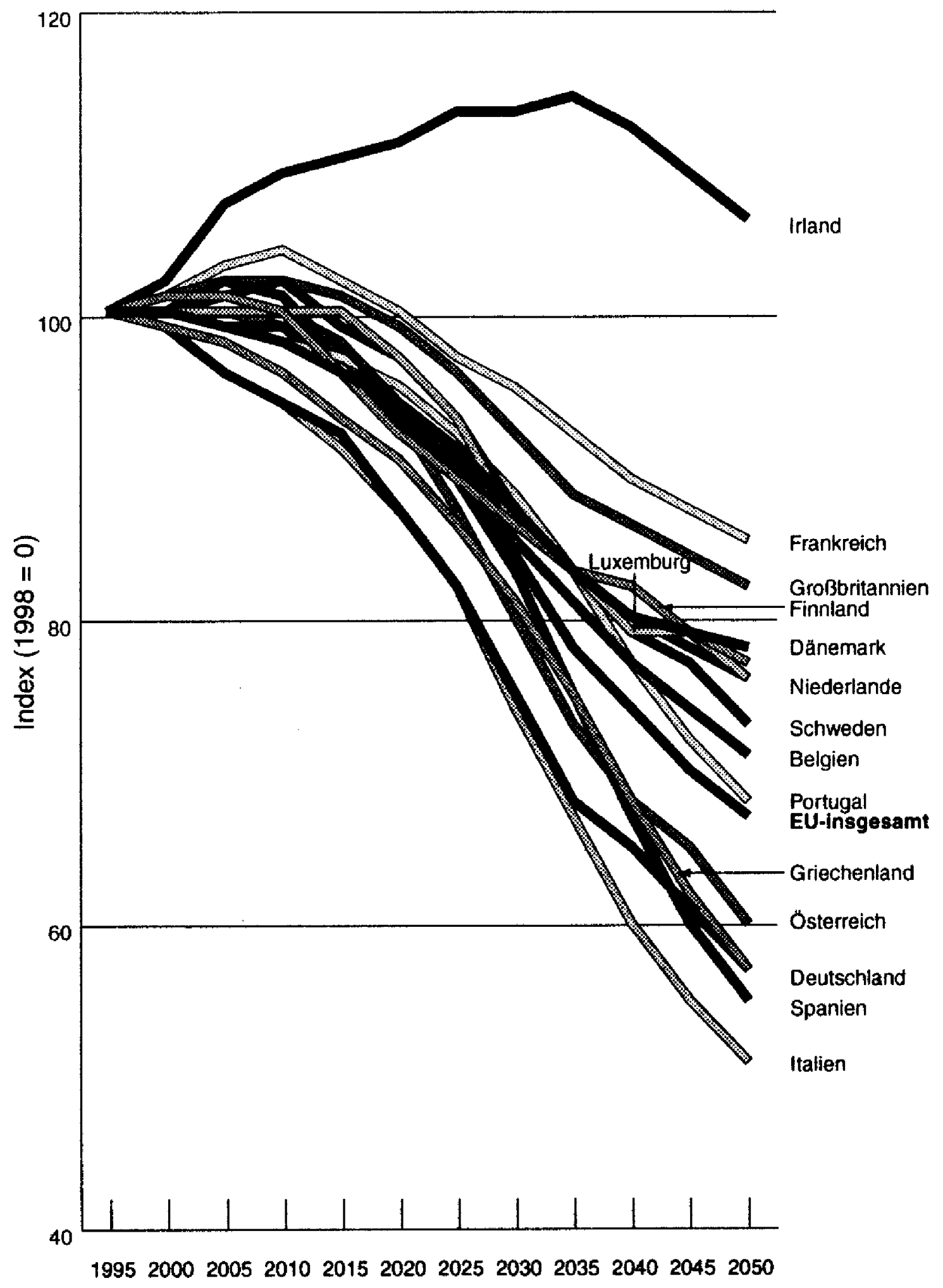

Quelle: H. Birg nach Berechnungen von Th. Frein, IBS, Universitäl Bielefeld 
Schaubild 10: Projektion der Zahl der Personen in der Altersgruppe 20 bis unter 40 in den Ländern der Europäischen Union, 1998-2050 (ohne Wanderungen)

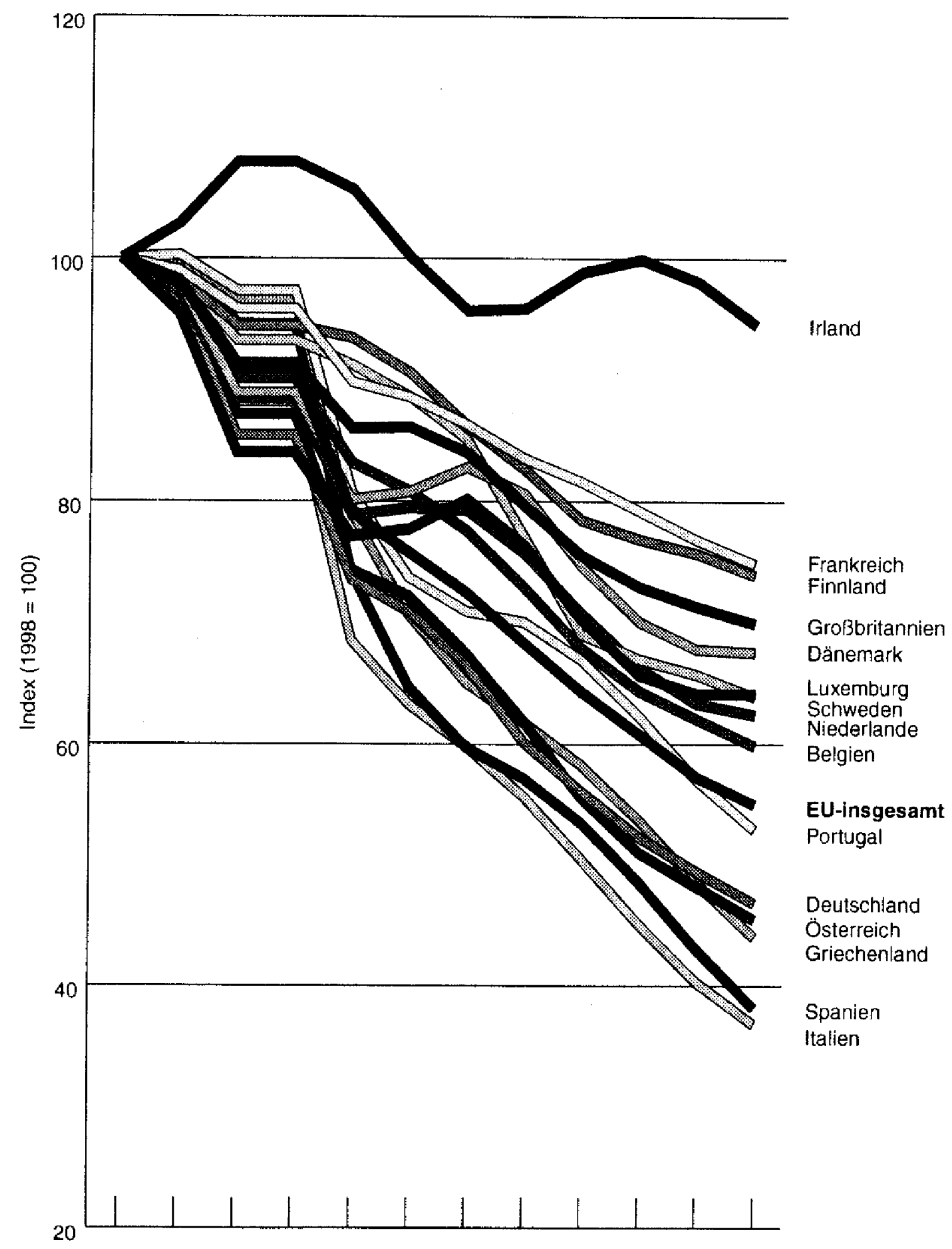

199520002005201020152020202520302035204020452050

Quelle: H. Birg nach Berechnungen von Th. Frein, IBS, Universität Bielefeld 
Aufgrund der überdurchschnittlichen Geburtenrate und der jungen Altersstruktur haben Frankreich und Großbritannien bis 2015 bzw. Irland bis 2050 auch ohne Einwanderungen noch Bevölkerungszuwächse, während die Bevölkerung in den übrigen EU-Ländern ohne Zuwanderungen bereits ab 1998 kontinuierlich zurückgeht. Aus den Daten und Analysen ergibt sich, dass ein Anstieg der Geburtenrate das wirksamste Mittel ist, um die Bevölkerungsschrumpfung langfristig zu stoppen und der demographischen Alterung der Gesellschaft entgegen zu wirken. Wollte man die demographische Alterung in der EU durch die Einwanderung jüngerer Menschen verhindern, müssten bis 2050 700,5 Mio. Menschen mehr ein- als auswandern, so dass die Bevölkerungszahl der EU von 1998 bis 2050 von 375 Mio. auf $1,2 \mathrm{Mrd}$. wachsen würde.

Diese Forschungsergebnisse zeigen, dass es absurd wäre, wenn eine demographisch orientierte Politik - statt eine Erhöhung der Geburtenrate anzustreben - auf Dauer auf eine zumindest teilweise Kompensation des Geburtendefizits durch Einwanderungen setzen würde, wie dies in Deutschland durch das Zuwanderungsgesetz geplant wird.

\section{Strategische Optionen der Familien- und Migrationspolitik am Beispiel Deutschlands}

Die folgenden Überlegungen beruhen auf Simulationsrechnungen für Deutschland, die nach alten und neuen Bundesländern getrennt durchgeführt wurden. Die Fertilitätsannahmen sind jeweils in drei Varianten untergliedert $(1,4$ bzw. 1,6 und 2,1 Lebendgeborene pro Frau). Bei der Zunahme der Lebenserwartung werden ebenfalls drei Varianten unterschieden (niedrig, mittel, hoch) und beim jährlichen Wanderungssaldo vier (Null-Variante, 150 Tsd., 225 Tsd. und 300 Tsd. pro Jahr). Die Kombination der Annahmen ergibt 36 Simulationsvarianten. ${ }^{5}$

Aus systematischen Gründen beginnt die folgende Darstellung der Ergebnisse mit einem Überblick über die Entwicklung der absoluten Bevölkerungszahl, obwohl die Entwicklung der demographischen Alterung und die Veränderung der für das Arbeitsangebot wichtigen Zahl der Personen im Alter 20-60 von größerer Bedeutung für die gesellschaftliche und wirtschaftliche Entwicklung sind. Bei einer unveränderten Fertilität von 1,4 Lebendgeborenen pro Frau und einem Anstieg der Lebenserwartung um 6 Jahre würde die Bevölkerungszahl Deutschlands ohne Wanderungen von 1995 bis 2050 von 82,0 Mio. auf 58,7 Mio. und bis 2100 auf 30,3 Mio. abnehmen. Bei einem allmählichen Anstieg der Kinderzahl pro Frau auf 1,6 (bis 2010) ergeben sich im Jahr 2050 63,9 Mio. statt 58,7 Mio. und im Jahr 2100 40,6 Mio. statt 30,3 Mio. Würde die Kinderzahl pro Frau bis 2030 allmählich auf das Bestandserhaltungsniveau von 2,1 zunehmen, betrüge die Bevölkerungs-

5 H. Birg/E.-J. Flöthmann/Th. Frein/K. Ströker: Simulationsrechnungen zur Bevölkerungsentwicklung in den alten und neuen Bundesländern im 2l. Jahrhundert. Materialien des Instituts für Bevölkerungsforschung und Sozialpolitik, Bd. 45, Universität Bielefeld, Bielefeld 1998. 
zahl im Jahr 2050 71,2 Mio. und im Jahr 2100 67,1 Mio. Bei einem Anstieg auf 2,1 Lebendgeborene pro Frau bis 2030 wäre die Zahl der Sterbefälle noch bis 2080 größer als die Zahl der Geburten, weil sich der Geburtenrückgang seit 1972 jetzt als Elternrlickgang auswirkt, der in Zukunft automatisch weitere Geburtenrïckgänge nach sich zieht usf. (Schaubild 11).

Wird der Anstieg der Kinderzahl pro Frau auf 1,6 mit einem jährlichen Wanderungsüberschuss von 150 Tsd. jungen Menschen kombiniert, ergibt sich eine gebrochene Entwicklung: Die Bevölkerungszahl nimmt bis 2010 auf $82,5 \mathrm{Mio}$. zu, danach geht sie bis 2050 auf 73,6 Mio. und bis 2100 auf 59,0 Mio. zurück. Bei einem höheren Wanderungssaldo von 225 Tsd. würde die Bevölkerungszahl bis 2020 auf $83,8 \mathrm{Mio}$. wachsen und sich anschließend bis 2050 auf 78,6 Mio. bzw. bis 2100 auf 68,4 Mio. verringern. Bei einem Wanderungssaldo von 300 Tsd. ergibt sich: Wachstum bis 2025 auf 85,8 Mio., danach Schrumpfung bis 2050 auf 83,6 Mio. bzw. bis 2100 auf 77,8 Mio.

Fazit: Durch einen Anstieg der Kinderzahl pro Frau von 1,4 auf 1,6, kombiniert mit einem jährlichen Wanderungssaldo von z. B. 150 Tsd. bzw. 225 Tsd., lässt sich die sonst zu erwartende Bevölkerungsschrumpfung bis 2010-2020 in ein leichtes Wachstum umkehren. Bei einem Wanderungssaldo von 300 Tsd. ergäbe sich ein Wachstum bis 2025, erst danach eine Schrumpfung.

Die Unterscheidung von zwei Phasen der Entwicklung vor und nach 2020 ist besonders wichtig für die Analyse der Entwicklung in der Altersgruppe 20-60, von der das Angebot an Erwerbspersonen entscheidend abhängt. Bei einer Geburtenrate von 1,4 Lebendgeborenen pro Frau und einem Wanderungssaldo von z.B. 150 Tsd. pro Jahr geht die Zahl der Personen in der Altersgruppe 20-60 in der ersten Phase von 2000-2020 noch relativ mäßig um 3,4 Mio. $(=-7,5 \%)$ zurück. Aber in der zweiten Phase von 2020-2050 beschleunigt sich der Rückgang stark, es ergibt sich eine zusätzliche Abnahme von weiteren 11,3 Mio. $(=-26,8 \%)$. Für beide Phasen zusammen (2000-2050) bedeutet dies eine Abnahme um 14,7 Mio. $(=32,3 \%)$. Gleichzeitig nimmt die Zahl der 60-Jährigen und älteren um 9 bis $10 \mathrm{Mio}$. zu, so dass sich der Altenquotient um den Faktor zwei bis drei erhöht.

Durch eine Zunahme der Geburtenrate von 1,4 auf beispielsweise 1,6 Lebendgeborene pro Frau wird der Rückgang des Erwerbspersonenpotentials in der ersten Phase von 2000-20 zwar nicht verringert, aber in der zweiten Phase von 2020-50 ergibt sich eine deutliche Abschwächung des Rückgangs. Da die Abnahme in der ersten Phase ohnehin vergleichsweise mäBig ist und durch arbeitsmarktpolitische Maßnahmen zur Verringerung der Arbeitslosigkeit weitgehend kompensiert werden kann, kommt es vor allem darauf an, die viel stärkere Abnahme in der zweiten Phase durch eine möglichst fruhzeitige Erhöhung der Geburtenrate zu mildern.

Wie stark sich eine Erhöhung der Geburtenrate auswirken würde, zeigen folgende Zahlen: Bei gleichbleibender Geburtenrate von 1,4 Kindern pro Frau sinkt die Zahl der Personen in der Altersgruppe 20-60 von 2020-2050 um 11,3 Mio. ( $=-26,8 \%$ ), bei einer Erhöhung von 1,4 auf 1,6 ergibt sich ein Rückgang um 8,7 Mio. $(=-20,7 \%)$, wobei in beiden Fällen von einem Wanderungssaldo von 150 Tsd. ausgegangen wurde (Schaubild 12). 


\section{Schaubild 11: Bevölkerungsprojektionen für Deutschland}
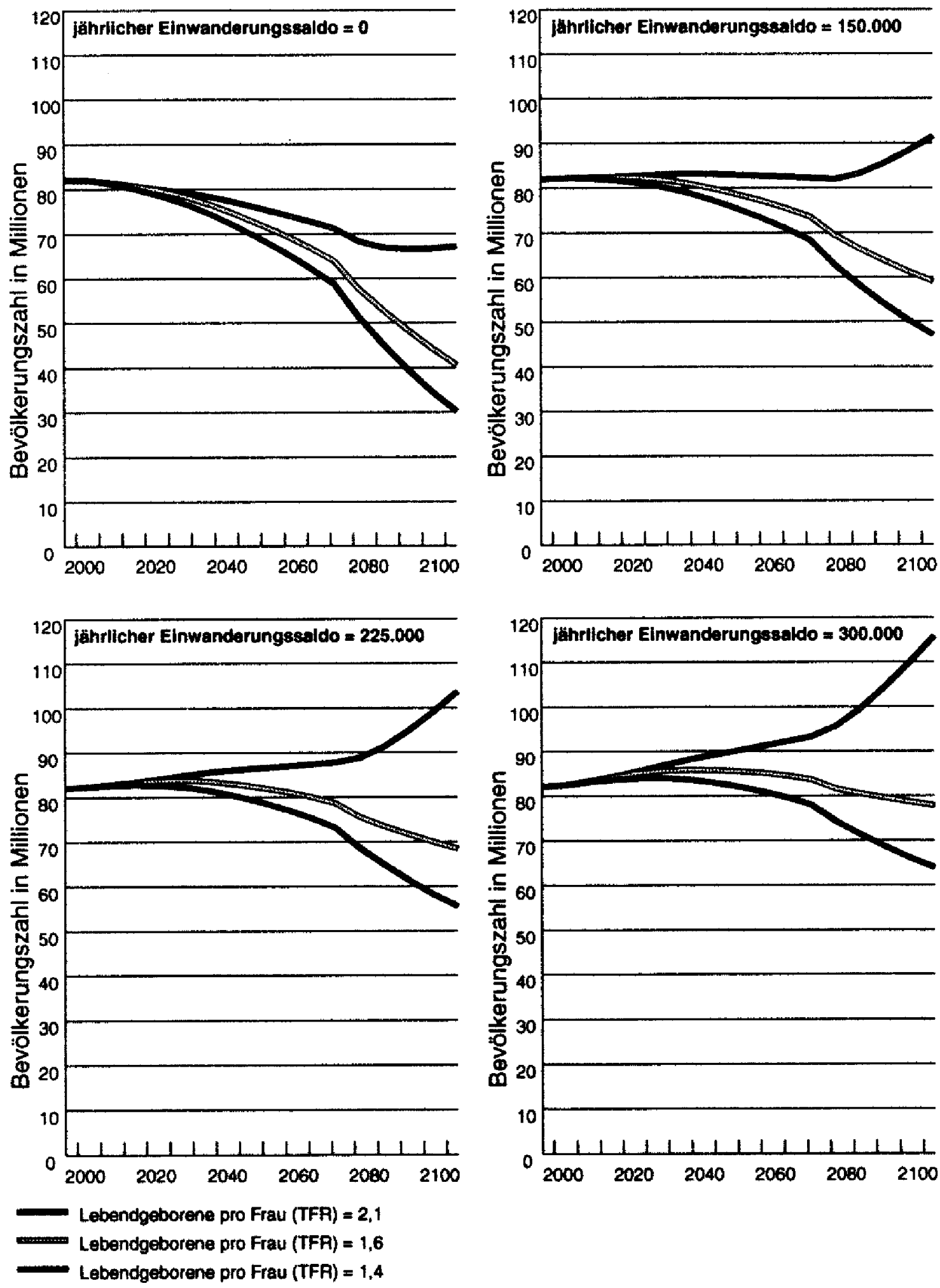

Quelle: Birg. H./FIothmann, E.-J./ Frein, T. u. Ströker, K.: Simulationsrechnungen der Bevolkerungsentwicklung in den alten und neuen Bundesländern im 21. Jahrhundert, Universitát Bieleleld, Bielefeld 1999 
Schaubild 12: Projektionsrechnung der Personen im erwerbsfähigen Alter
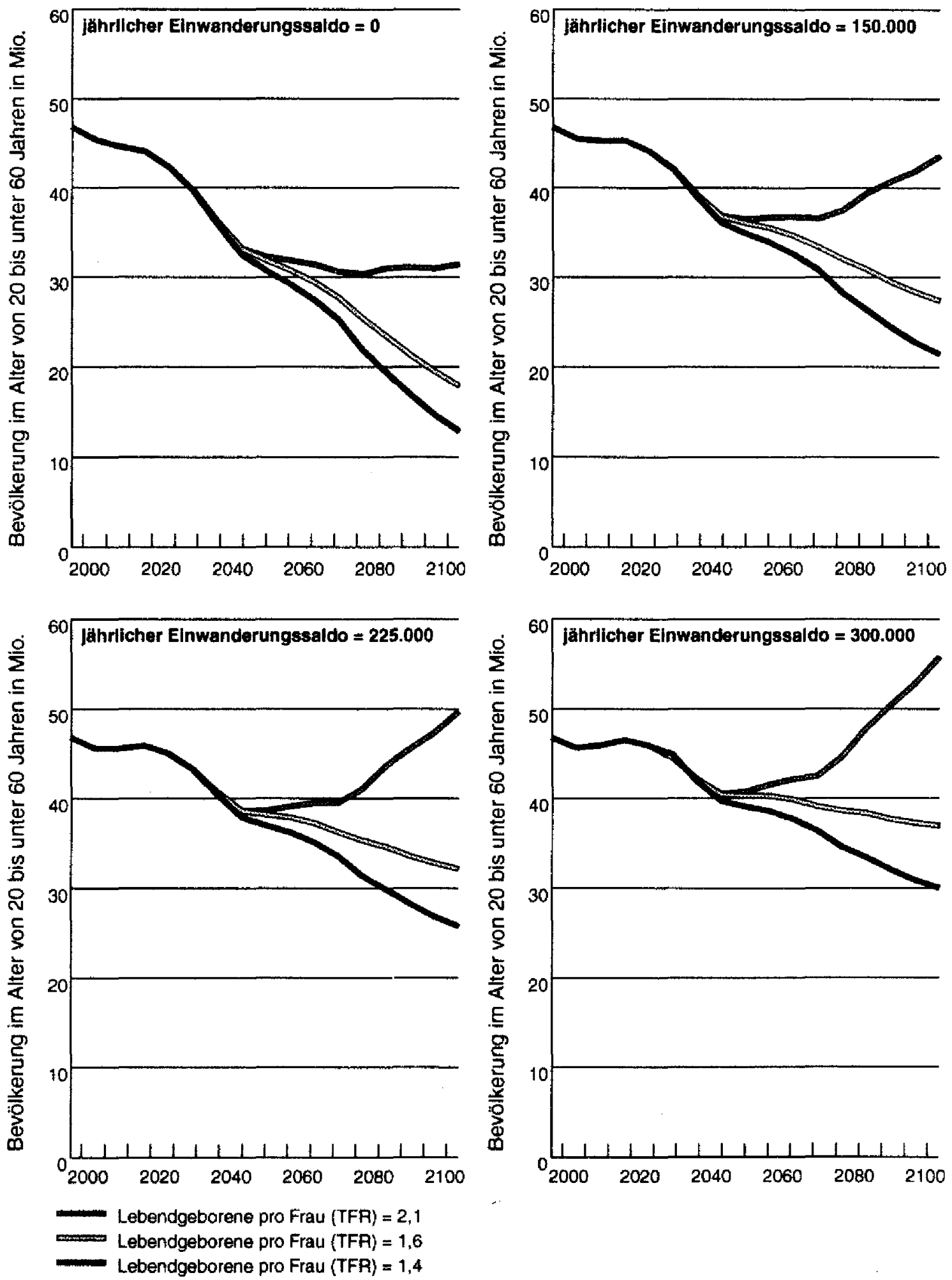

Quelle: Birg, H./FIöthmann, E.-J./ Frein, T. u. Ströker, K.: Simulationsrechnungen der Bevolkerungsentwicklung in den alten und neven Bundesländern im 21. Jahrhundert, Universität Bielefeld, Bielefeld 1999 
Fazit: Erfolge in der Familienpolitik durch eine Erhöhung der Geburtenrate schlagen nach 20 Jahren als Erfolge auf dem Arbeitsmarkt zu Buche. Ein Anstieg der Geburtenrate von 1,4 auf 1,6 Lebendgeborene pro Frau würde den Rückgang des Arbeitsangebots von 2020-2050 um 2,6 Mio. mildern.

Die Stärkung der Familienpolitik mit dem Ziel einer Erhöhung der Geburtenrate zur Stützung des Arbeitsangebots ist eine strategische Option der Politik, die gegenüber der bisher praktizierten Strategie der Kompensation der demographischen Defizite durch Einwanderungen entscheidende Vorteile hat. Der erste Vorteil besteht darin, dass sie die Kosten der Integration reduziert. Wie die vom Ifo-Institut und vom Max-Planck-Institut für ausländisches und internationales Sozialrecht im Auftrag des Bundesarbeitsministeriums durchgeführten Forschungsarbeiten zeigen, übersteigen die vom Staat für die Zugewanderten erbrachten fiskalischen Leistungen im Rahmen der Sozialversicherung (gesetzliche Krankenversicherung, Renten- und Pflegeversicherung) sowie die steuerfinanzierten Transfers und die Zahlungen der Gebietskörperschaften für die Bereitstellung der öffentlichen Güter (Schulen, Krankenhäuser, Verkehrsinfrastruktur, Verwaltung etc.) die vom Staat von den Zugewanderten empfangenen Leistungen pro Kopf und Jahr um mehrere Tausend DM (Tabelle 3). Dieser Befund widerspricht den landläufigen Vorstellungen, dass Deutschland fiskalisch von der Zuwanderung profitiert. Nach dieser Untersuchung war und ist die Zuwanderung nach Deutschland seit langem eine „Zuwanderung in die Sozialsysteme", die eine „Umverteilung von den Deutschen zu den Zugewanderten" bewirkt, wie es in dem Forschungsbericht heißt. ${ }^{6}$

Der zweite wesentliche Vorteil der familienpolitischen Strategie gegenüber einer Strategie der kompensatorischen Zuwanderung besteht in einer Stärkung des Wachstums des Pro-Kopf-Einkommens. Ein befriedigendes Wachstum der Produktivität und des Pro-Kopf-Einkommens setzt ein hohes Qualifikationsniveau der Arbeitskräfte voraus. Das durchschnittliche Qualifikationsniveau der nach Deutschland zugewanderten Bevölkerung ist im Vergleich zum durchschnittlichen Qualifikationsniveau der deutschen Bevölkerung wesentlich niedriger. Von den ausländischen Schulabgängern in Deutschland im Jahr 1999 hatten z.B. 40,9\% einen Hauptschulabschluss (Deutsche: $24,7 \%$ ) und $19,4 \%$ verließen das Schulsystem ohne Abschluss (Deutsche: $8,0 \%$ ).

Die Qualifikationsdefizite sind der entscheidende Grund dafür, dass die Arbeitslosenquote und die Quote der Sozialhilfeempfänger bei den Zugewanderten aus Nicht-EU-Ländern um den Faktor 3 und mehr höher ist als bei den Einheimischen, und zwar nicht nur in Deutschland, sondern in nahezu allen Ländern der EU (Schaubild 13). Die Qualifikationsdefizite sind dabei um so größer, je höher der Anteil der Zugewanderten an der Bevölkerung ist (Schaubild 14). Aufgrund dieser

6 H.-W. Sinn u.a.: EU-Erweiterung und Arbeitskräftemigration - Wege zu einer schrittweisen Annäherung der Arbeitsmärkte. Ifo-Beiträge zur Wirtschaftsforschung, Nr. 2, München 2001, S. 225-27.

7 Deutsches Institut für Wirtschaftsforschung (Hrsg.): Schulbesuch und Ausbildung von jungen Ausländern, Wochenbericht Nr. 10/2001, Tabelle 1, S. 163 u. Tabelle 3, S. 166. 
Tabelle 3: Direkte fiskalische Auswirkungen der Zuwanderung pro Zuwanderer $^{1}$ - Westdeutschland 1997 (in DM)

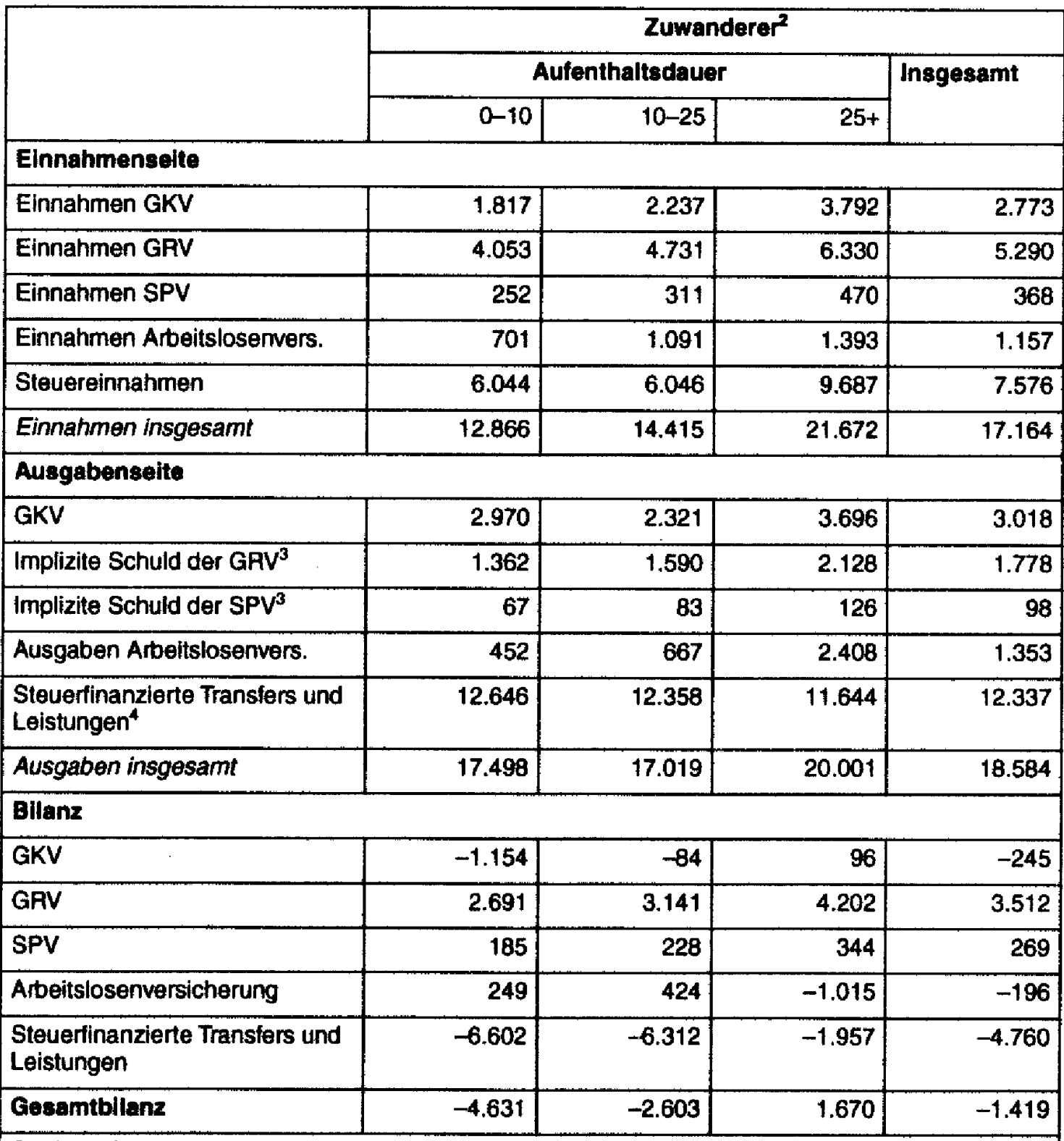

Quelle: SOEP; Berechnungen des Ifo-Instituts. In: W. Sinn u.a., EU-Erweiterung und Arbeitskrăftemigration, Ifo-Institut in Zusammenarbeit mit dem Max-Planck-tnstitut für ausländisches und internationales Sozjairecht, lio-Beiträge zur Wirtschaftsforschung, Nr. 2, München 2001, S. 227.

1. Die Zahlen der vorliegenden Tabelle spiegeln ausschließlich die relative Position derjenigen Zuwanderer wider, die sich im Jahr 1997 in Westdeutschland befanden. Eine direkte Übertragung auf die zu erwartenden Zuwanderer aus den osteuropäischen Lăndem ist nicht zulässig, da sich die Struktur zukünftiger Einwanderungskohorten von der des Zuwandererbestandes 1997 unterscheiden dürte.

2. Zuwanderer umfassen in Deutschland lebende Personen mit nicht-deutscher Nationalität, aber auch in Deutschland eingebürgerte Personen und Personen mit Müttern nicht-deutscher Nationalităt ohne die Gruppe der Aus- und Übersiedler.

3. Die Berechnung der GRV- und Pflegeausgaben entolgt nach dem Konzept der Impliziten Steuer.

4. Leistungen der Gebietsköperschaften an private Haushalte und Durchschnittskosten der Bereitstellung Offentlicher Güter. 
Fakten ist auch in Zukunft nicht damit zu rechnen, dass die Qualifikationsunterschiede im erhofften Umfang abgebaut werden können.

Durch die Strategie einer kompensatorischen Zuwanderungspolitik würde das für die Produktivität und das Pro-Kopf-Einkommen wichtige, im Humankapital der jüngeren Erwerbspersonen enthaltene Bildungs- und Ausbildungskapital beeinträchtigt. Die Strategie höherer Zuwanderungen hat zwar naturgemäß in aller Regel eine höhere Arbeitskräftezahl und dadurch ein höheres Bruttosozialprodukt zur Folge als die alternative Strategie geringerer Zuwanderungen und einer verstärkten Familienpolitik, aber die Höhe des Pro-Kopf-Einkommens, auf die es ankommt, ist bei mäßigeren Zuwanderungen wahrscheinlich günstiger als bei hohen Zuwanderungen, wie folgende Beispielrechnung zeigt: ${ }^{8}$

Strategie hoher Zuwanderungen:

Wachstumsrate des Bruttosozialprodukts

$=2,5 \%$

Wachstumsrate der Bevölkerung

Wachstumsrate des Pro-Kopf-Einkommens $(2,5-0,7=1,8)$

$=0,7 \%$

$=1,8 \%$

Strategie mäßiger Zuwanderung:

Wachstumsrate des Bruttosozialprodukts

$=1,7 \%$

Wachstumsrate der Bevölkerung

$=-0,7 \%$

Wachstumsrate des Pro-Kopf-Einkommens $(1,7-(-0,7)=2,4)$

$=2,4 \%$

In der öffentlichen Debatte über die Zuwanderungen nach Deutschland wird stets der positive Effekt der Zuwanderungen auf die Höhe des Bruttosozialprodukts herausgestellt. Aber es kommt auf die Höhe des Pro-Kopf-Bruttosozialprodukts an. Die Schweiz übt nicht deshalb eine magnetische Anziehungskraft auf die Zuwanderer z.B. aus den Entwicklungsländem Asiens aus, weil das Bruttosozialprodukt der Schweiz größer wäre als das Bruttosozialprodukt Asiens, sondern weil das Pro-Kopf-Bruttosozialprodukt und der mit ihm korrelierende Lebensstandard in der Schweiz wesentlich höher ist.

\section{Resümee}

In den letzten 50 Jahren gingen die Geburtenraten in den Industrieländern um etwa die Hälfte zurück. In Deutschland beruhte der Rückgang vor allem auf dem Anstieg des Anteils der Frauen an einem Jahrgang mit lebenslanger Kinderlosigkeit auf rd. ein Drittel, während sich bei den Frauen mit Kindern nach wie vor eine im langfristigen Vergleich konstante Zahl von rd. zwei Kindern ergibt. Bei den EULändern, bei denen der Anteil kinderloser Frauen niedrig ist (z.B. Frankreich),

8 Die Wachstumsrate des Pro-Kopf-Einkommens ist mathematisch gleich der Differenz zwischen den Wachstumsraten des Bruttosozialprodukts und der Bevölkerung. 
Schaubild 13: Arbeitsmarktintegration von Zuwanderern

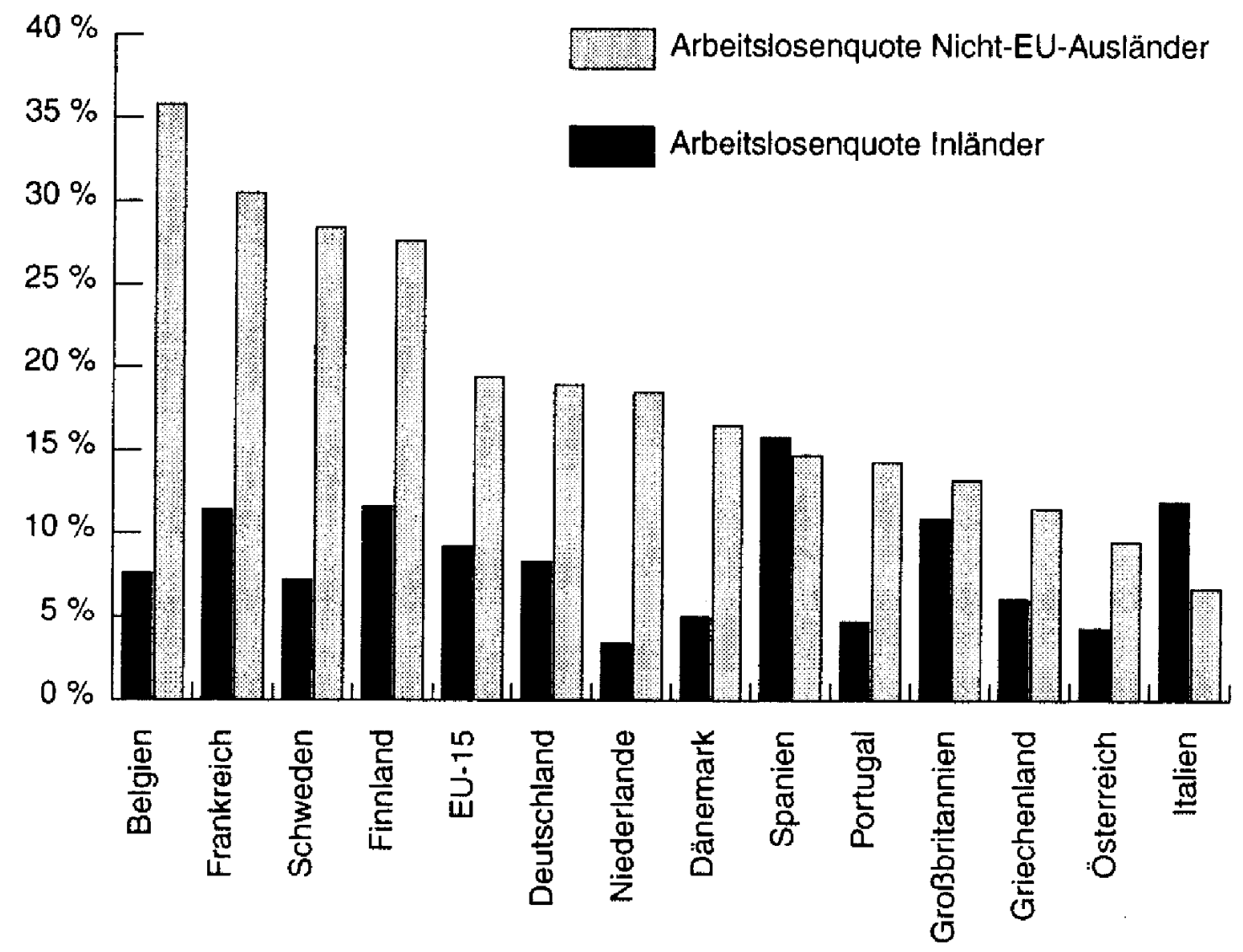

Quelle: U. van Suntum \& D. Schlotböller: Arbeitsmarktintegration von Zuwanderern, Gütersloh 2002.

liegt die Geburtenrate über dem Durchschnitt der EU, bei Ländern mit hoher Kinderlosigkeit unter dem Durchschnitt (z.B. Deutschland).

Durch die in Deutschland besonders hohe Kinderlosigkeit spaltet sich die Gesellschaft in einen Familiensektor und in einen Sektor ohne eigene Nachkommen. Daraus ergeben sich gravierende Konsequenzen für das in der Verfassung verankerte Prinzip der sozialen Gerechtigkeit, durch dessen Verletzung auch die sozialen Sicherungssysteme ihre Funktion nicht mehr erfüllen können.

Deutschland betreibt seit dem Zweiten Weltkrieg keine Bevölkerungspolitik mehr - mit einer wesentlichen Ausnahme: Die Zuwanderungspolitik in Deutschland ist eine kompensatorische Bevölkerungspolitik, mit der ein immer größerer Anteil der durch Tod ausgeschiedenen Generationen durch Zuwanderungen aus dem Ausland ersetzt statt durch Geburten erneuert wird.

Der Generationenersatz durch Zuwanderungen ist sowohl aus wirtschaftlicher als auch aus gesellschaftspolitischer Sicht ungünstiger als die alternative Strategie einer Generationenerneuerung durch eine Erhöhung der Geburtenrate. Bei hohen Zuwanderungen verringert sich das Qualifikationsniveau der Bevölkerung, und es kommt zu Einbußen beim Wachstum des Pro-Kopf-Einkommens, während gleichzeitig die Integrationskosten steigen. 
Schaubild 14: Bevölkerungsanteil und Bildungsrïckstand von Nicht-EU-Bürgern (Jahresmittel 1992-1999)

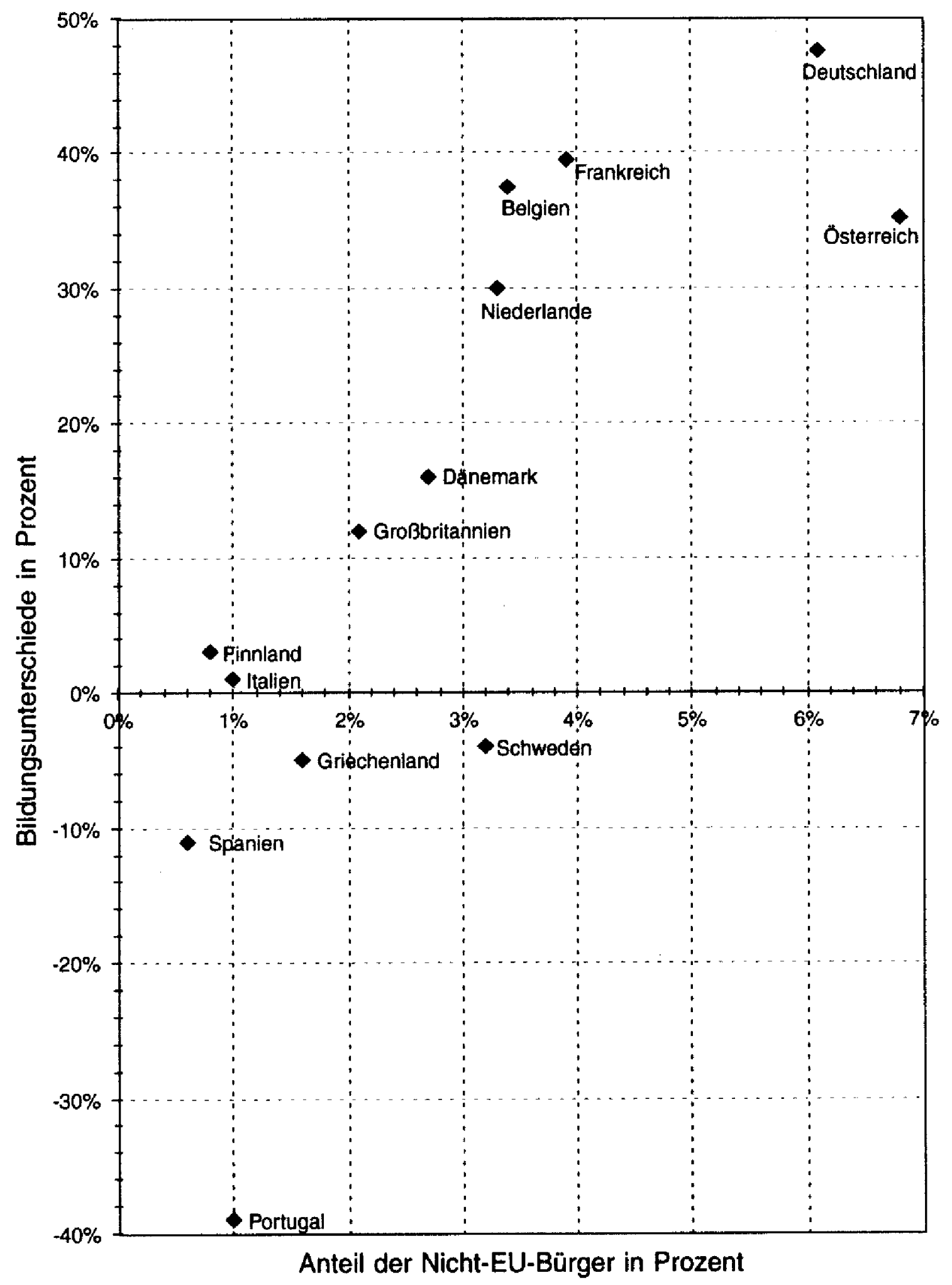

Quelle: U. van Suntum \& D. Schlotböller: Arbeitsmarktintegration von Zuwanderern, Gütersloh 2002 
Die Strategie des Generationenersatzes durch Zuwanderungen ist auch aus internationaler Sicht problematisch. Denn wenn Zuwanderungen für das aufnehmende Land ökonomische Vorteile bringen, drohen diese Vorteile den Herkunftsländern verloren zu gehen. Entscheidend ist jedoch, dass auch Einwanderer geboren, erzogen und ausgebildet werden müssen, bevor sie im Wettbewerb um die Besten in ein anderes Land einwandern können. Es wäre eine moralisch durch nichts zu rechtfertigende Strategie, wenn die reichen Länder auf Dauer ihre demographischen Defizite auf Kosten der armen ausgleichen und mit den Mitteln der Migrationspolitik eine Art demographischen Kolonialismus etablieren würden.

\section{Quellen}

H. Birg: Auswirkungen und Kosten der Zuwanderung nach Deutschland. Gutachten im Auftrag des Bayerischen Staatsministeriums des Innern. Materialien des Instituts für Bevölkerungsforschung und Sozialpolitik, Bd. 49, Universität Bielefeld, Bielefeld 2002.

H. Birg und E.-J. Flöthmann: Demographische Projektionsrechnungen für die Rentenreform 2000 - Methodischer Ansatz und Hauptergebnisse. Materialien des Instituts für Bevölkerungsforschung und Sozialpolitik, Bd. 47A, Universität Bielefeld, Bielefeld 200I

H. Birg und E.-J. Flöthmann: Demographische Projektionsrechnungen für die Rentenreform 2000 - Ergebnisse in Tabellenform. Materialien des Instituts für Bevölkerungsforschung und Sozialpolitik, Bd. 47B, Universität Bielefeld, Bielefeld 2001.

H. Birg: Die demographische Zeitenwende - Der Bevölkerungsrïckgang in Deutschland und Europa. Beck'sche Reihe, Verlag C.H. Beck, München 2001.

Bundesministerium des Innern/Unabhängige Kommission für Zuwanderung (Hrsg.): Zuwanderung gestalten - Integration fördern, Berlin, 4. Juli 2001.

D. Coleman: Migration nach Europa. Eine Kritik am neuen Konsens des Establishments. In: Zeitschrift für Bevölkerungswissenschaft, 26. Jg., Nr. 3-4, 2001, S. 327-340.

Deutscher Bundestag (Hrsg.): Schlussbericht der Enquete-Kommission des Deutschen Bundestages "Demographischer Wandel", Bundestagsdrucksache 14/8800, Berlin, 28.3.2002.

Deutsches Institut für Wirtschaftsforschung (Hrsg.): Schulbesuch und Ausbildung von jungen Ausländern, Wochenbericht Nr. 10/2001.

H.-W. Sinn u. a.: EU-Erweiterung und Arbeitskräftemigration - Wege zu einer schrittweisen Annäherung der Arbeitsmärkte. lfo-Beiträge zur Wirtschaftsforschung, Nr. 2, München 2001.

U. van Suntum u. D. Schlotböller: Arbeitsmarktintegration von Zuwanderern, Gütersloh 2002.

Statistisches Bundesamt (Hrsg.): Bevölkerungsentwicklung Deutschlands bis zum Jahr $2050-E r$. gebnisse der 9. koordinierten Bevölkerungsvorausberechnung, Wiesbaden 2000.

UN (Ed.): Replacement migration: Is it a solution to declining and ageing populations? Population Division, Dept. of Economic and Social Affairs, United Nations Secretariat, New York 2000.

UN (Ed.): World Population Prospects - The 2000 Revision, New York 2001. 